\title{
MIMO Exploitation of 3D Multipath Statistics in a Heterogeneous LTE-Advanced Network
}

\author{
Zuhanis Mansor, Evangelos Mellios, Geoffrey Hilton, Joe McGeehan, and Andrew Nix
}

Communication Systems \& Networks Group, Department of Electrical \& Electronic Engineering,

Merchant Venturers Building, Woodland Road, Bristol BS8 1UB, UK

Correspondence should be addressed to Zuhanis Mansor; anis.mansor@bristol.ac.uk

Received 31 March 2013; Revised 12 June 2013; Accepted 14 June 2013

Academic Editor: Yan Zhang

Copyright (c) 2013 Zuhanis Mansor et al. This is an open access article distributed under the Creative Commons Attribution License, which permits unrestricted use, distribution, and reproduction in any medium, provided the original work is properly cited.

\begin{abstract}
This paper analyses the impact of 3D multipath in an LTE-Advanced heterogeneous network. The impact of the base station array configuration is considered in both the azimuth and elevation planes. Spatial and temporal multipath statistics are generated for example macro- and picocellular base stations, and these are combined with appropriately oriented complex polarimetric antenna patterns. The resulting wideband channels are then passed to an LTE-Advanced physical layer simulator. The optimal spatial multiplexing mode is determined by computing the throughput using the received bit mutual information rate for all modulation and coding schemes. The fastest link speed for each user is identified given a packet error rate threshold of $10 \%$. Results show that RMS angle spread statistics and the base station MIMO array configuration strongly influence user performance in an LTE-A network. The deployment of macrocellular vertical arrays is shown to significantly degrade network performance. In contrast, the elevation angle spread is found to be far greater in picocells, and this allows vertical arrays to be applied to produce compact high performance picobase stations.
\end{abstract}

\section{Introduction}

The growth of smart handsets has led to mobile users demanding higher data rates and better quality mobile services. Traditional wireless telecommunication systems no longer meet the aggregate traffic demands of the user base. In order to meet the needs of next generation networks, such as LTE-Advanced (LTE-A) [1], the spectrum efficiency of future mobile communication systems must be improved. This can be achieved by adopting advanced technologies such as multiple-input multiple-output (MIMO) antenna arrays and heterogeneous networks (HetNets). Developed by 3GPP, LTE-A is an evolved version of LTE. LTE-A meets the requirement of the International Telecommunication Union (ITU) for classification as a fourth generation (4G) radio standard. LTE-A makes use of carrier aggregation, advanced MIMO techniques, wireless relays, intercell interference coordination (ICIC), and coordinated multipoint (CoMP) transmission/reception. LTE-A targets low mobility (pedestrian) users at velocities up to $15 \mathrm{~km} / \mathrm{h}$.
Considerable work has been published on MIMO performance in an LTE-A network by Prasad et al. and Li et al. $[2,3]$. Lee et al. in [4] discussed the MIMO technologies in 3GPP LTE and LTE-A. The work focused on average cell throughput and cell-edge user throughputs on the downlink. Results showed that for $2 \times 2$ and $4 \times 4$ antenna configurations the use of spatial multiplexing (SM) improved the average cell throughput. SM increases the user data rate by transmitting independent data streams simultaneously over unique spatial channels. Gains in average cell throughput of $8.2 \%$ have been reported for a $4 \times 2$ MIMO configuration compared to a $2 \times 2$ reference network. MIMO technologies were also shown to significantly improve cell-edge user throughput.

This paper analyses the performance of the MIMO techniques that enable LTE-A to achieve a peak spectral efficiency of $30 \mathrm{bps} / \mathrm{Hz}$ on the downlink (DL) and $15 \mathrm{bps} / \mathrm{Hz}$ on the uplink (UL). In particular, this paper quantifies the relationship between the MIMO data rates and 3D multipath angle statistics at the base station and mobile terminals. According to the mobile data traffic statistics reported by 

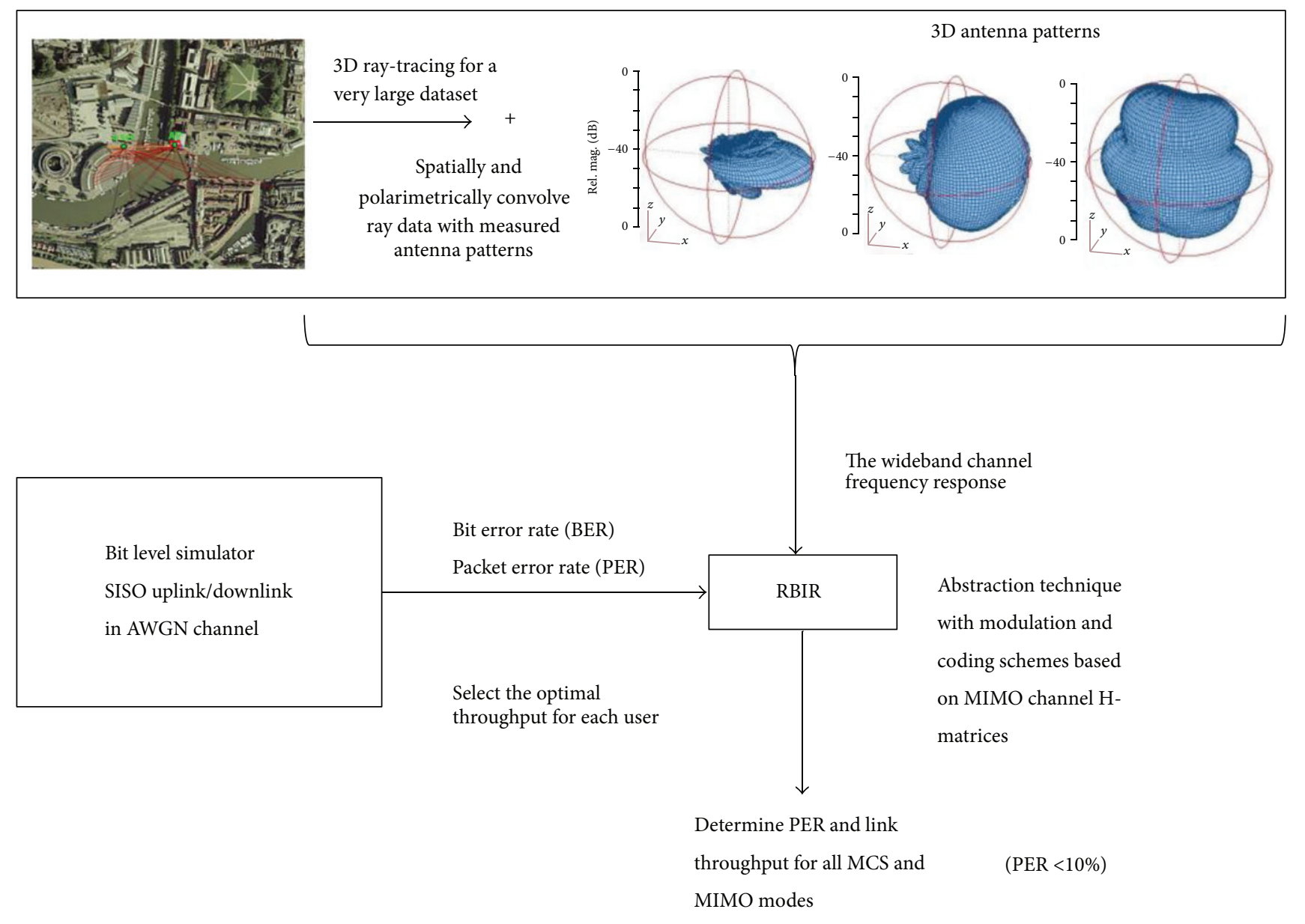

FIGURE 1: Graphical summary of the modelling methodology.

Cisco Visual Networking Index in [5], mobile data traffic is expected to grow at a compound annual growth rate (CAGR) of $92 \%$ from 2010 to 2015, reaching 6.3 exabytes per month by 2015 . In order to enhance the peak data rates as well as the overall network capacity, one interesting solution is to complement the macrolayer with a number of low power picobase stations. The resulting solution is a HetNet [69]. HetNets are a new technique for increasing the capacity of LTE-A networks. The initial deployments of LTE consist of macrobase stations and user equipment (UE) terminals that cater for high mobility users. However, HetNets use a combination of macro- and picobase stations to increase the spectral efficiency per unit area. Khandekar et al. in [6] discussed the use of HetNets to enhance range.

Few papers to date have analysed the throughput performance of LTE-A users in a picocellular scenario. HetNets allow many users to associate with the pico base stations and this enables a more equitable per user distribution of the radio resources, especially in regions with hotspots. In [7] a number of picocells were deployed at the edge of the macrocell to mitigate coverage holes. Previous work by Landström et al. in [8] has studied the position of the picocells relative to the macrosites. It was found that the location of the picocells has a significant impact on capacity. The performance of three different HetNet deployments was compared by Hiltunen in [10]. The results indicated that HetNet deployments are valuable alternatives to traditional macrosites.

The impact of user handset antenna pattern and orientation was investigated by Mansor et al. [11] in a singleinput single-output (SISO) HetNet scenario with a single base station and six users. This paper expands on [11] by considering a HetNet deployment for $2 \times 2$ MIMO links with different base station array configurations for a very large number of links (7,283 pico base station-UE links and 4,076 macro base station-UE links). All analyses are performed in $3 \mathrm{D}$ space. The urban ray-tracing channels described in this paper have been used as the basis of a proposed 3D extension of the 3GPP/ITU channel model [12-14]. Figure 1 illustrates a graphical representation of the simulation methodology used in this paper.

$3 \mathrm{D}$ ray tracing is used to model all the significant multipath components between the base station and mobile terminal given the 3D geometry of the base station and mobile arrays. Measured 3D complex field antenna patterns are then spatially and polarimetrically convolved with a set of 1000 channels generated from the original ray data. The resulting channel impulse response for each link is then transformed into the frequency domain to determine the wideband 
TABLE 1: Parameters for LTE-A OFDMA.

\begin{tabular}{ll}
\hline System parameters & Specifications \\
\hline Carrier frequency & $2.6 \mathrm{GHz}$ \\
Transmission bandwidth & $10 \mathrm{MHz}$ \\
Time slot/subframe duration & $0.5 \mathrm{~ms} / 1 \mathrm{~ms}$ \\
Subcarrier spacing & $15 \mathrm{kHz}$ \\
Sampling frequency & $15.36 \mathrm{MHz}(4 \times 3.84 \mathrm{MHz})$ \\
IFFT size & 1024 \\
Number of occupied subcarrier & 600 \\
Number of OFDMA symbols & 7 \\
per time slot (short CP) & $(4.69 / 72) \times 6,(5.21 / 80) \times 1$ \\
CP length ( $\mu$ s/samples) & Perfect \\
Channel knowledge & Distributed \\
Subcarrier mapping scheme & Turbo \\
Channel coding & $2 \times 2$ \\
$N_{\text {SS }} N_{\text {UE }}$ & SM (closed-loop) \\
MIMO & Pico: $\lambda, 2 \lambda, 3 \lambda$ \\
BS antenna spacing (Figure 3) & Macro: $3 \lambda, 5 \lambda, 10 \lambda$ \\
UE antenna spacing (Figure 3) & $0.5 \lambda, \lambda, 2 \lambda$ \\
\hline
\end{tabular}

channel response. As shown in the left-hand side of Figure 1, a bit accurate LTE-A physical layer simulator is used to determine the single antenna AWGN packet error rate (PER) versus signal-to-noise ratio (SNR) graph for each Modulation and coding scheme (MCS). The AWGN graphs are then passed along with the set of 1000 wideband channels into the received bit mutual information rate (RBIR) simulator to determine the instantaneous packet error rate (PER) for all MIMO MCS modes at the SNR determined from the raytracing data. The MCS mode that maximises the throughput for each link while maintaining a PER $<10 \%$ is then chosen by the link adaptation algorithm. Finally, statistics are generated based on the MCS modes and data throughputs of the 11,000+ links.

This work exploits the novel combination of MIMO LTEAdvanced base station geometries, detailed $3 \mathrm{D}$ ray-tracing data sets, measured 3D antenna patterns, and advanced physical layer simulation and link speed selection algorithms. In particular, for urban $2.6 \mathrm{GHz}$ pico- and macrocellular MIMO deployments, the paper makes the following key contributions.

(1) A detailed analysis of the 3D multipath statistics based on more than 11,000 ray-traced links. For each link the multipath data is spatially and polarimetrically convolved with measured $3 \mathrm{D}$ antenna patterns to form an integrated antenna/channel model.

(2) The end-to-end performance of horizontally and vertically oriented MIMO base station antenna arrays is investigated for LTE-A. Results are compared with the azimuth, and elevation RMS angle spread results in both cell types.
(3) An analysis of the eigenvalue spread of the MIMO channel and its relationship to the optimal LTE-A link speed.

(4) An investigation into the likelihood (occurrence rate) of selecting a particular MIMO enabled MCS mode as a function of cell type, antenna configuration and inter-element spacing.

The outline of this paper is as follows. Section 2 presents the key parameters and assumptions behind the MIMO channel model. Section 3 describes the propagation channel model and the antenna radiation patterns used in this study. It also includes a statistical analysis of the RMS delay spread, $\mathrm{K}$-factor, and 3D arrival and departure angle statistics for urban pico- and macrocellular deployments. Results focused on LTE-A performance in both cell types using vertical and horizontal base station antenna arrays (for various antenna spacings) are reported in Section 4. Metrics based on PER, throughput, and occurrence rate for specific MCS modes are presented, and these are linked to the RMS angle spread and eigenvalue spread statistics of the channel correlation matrix. Finally, conclusions are drawn in Section 6.

\section{MIMO Channel Model}

The performance of MIMO is critically dependent on the availability of independent spatial channels. It is well-known that channel correlation degrades the performance of a MIMO system. Spatial correlation is dependent on the $3 \mathrm{D}$ multipath structure. Multipath signals depart from the base station with a given Angle of Departure (AoD). At the mobile terminal, the multipath signals arrive via a unique set of Angle of Arrivals (AoA). Spatial correlation in the horizontal plane is related to the azimuth angle spread, while spatial 
TABLE 2: MCS PHY layer in $10 \mathrm{MHz}$ bandwidth.

\begin{tabular}{lccccc}
\hline MCS & Modulation & Coding rate, $r_{c}$ & $\begin{array}{c}\text { Coded bits per } \\
\text { subcarrier, } b\end{array}$ & $\begin{array}{c}\text { Data bits per subcarrier } \\
\text { per time slot }(0.5 \mathrm{~ms}) \\
(2 \times 2)\end{array}$ & $\begin{array}{c}\text { Nominal bit rate }(\mathrm{Mbps}) \\
(2 \times 2)\end{array}$ \\
\hline 1 & QPSK & $1 / 2$ & 2 & 8400 & 16.80 \\
2 & QPSK & $3 / 4$ & 2 & 12600 & 25.20 \\
3 & $16-$ QAM & $1 / 2$ & 4 & 16800 & 33.60 \\
4 & 16-QAM & $3 / 5$ & 4 & 20160 & 40.32 \\
5 & 64-QAM & $1 / 2$ & 6 & 25200 & 50.42 \\
6 & 64-QAM & $2 / 3$ & 6 & 33600 & 60.72 \\
7 & 64-QAM & $3 / 4$ & 6 & 37800 & 75.60 \\
\hline
\end{tabular}

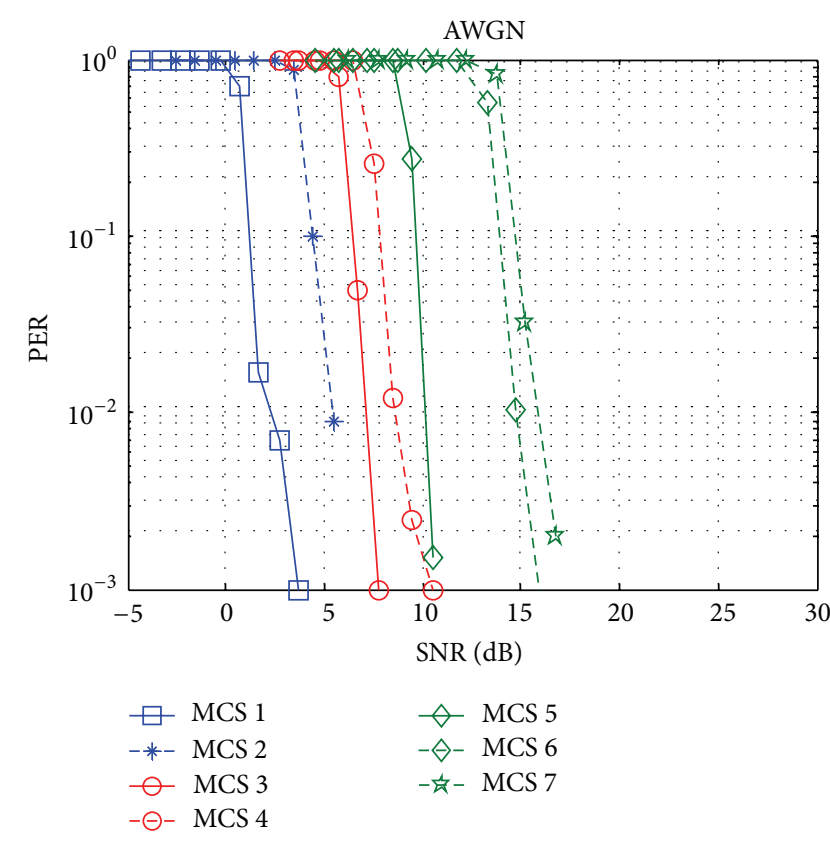

(a)

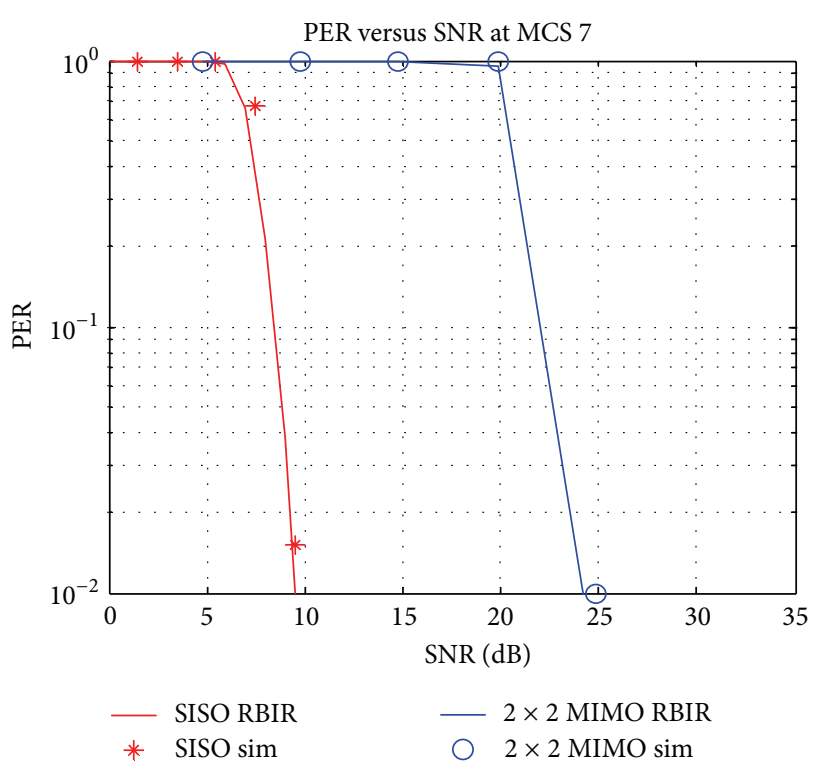

(b)

FIGURE 2: OFDMA for (a) PER under ideal AWGN channel conditions and (b) validation of RBIR abstraction for a wideband channel $(2 \times 2$ SM with MCS mode 7).

correlation in the vertical plane is related to the elevation angle spread. Current models assume propagation in a $2 \mathrm{D}$ horizontal plane. However, recent work has proposed 3D extensions to the 3GPP/ITU channel model [12-14]. This paper exploits these new $3 \mathrm{D}$ channel models to investigate the viability of vertically spaced MIMO arrays at the base station. Results for macro- and picocells are presented and compared against traditional horizontally spaced MIMO arrays.

\section{System Design and Parameters}

The LTE-A downlink for $2 \times 2$ MIMO with two transmit and two receive antennas is studied using a baseband link-level simulator. Table 1 shows the key parameters of the LTE-A FDD downlink simulation. We assume a channel bandwidth of $10 \mathrm{MHz}$ and a carrier frequency of $2.6 \mathrm{GHz}$. All physical layer parameters, unless explicitly stated, are listed in Table 1.
A receive noise figure (NF) of $5 \mathrm{~dB}$ was assumed at each base station [9]. The PER for each of the MIMO OFDMA PHY layer transmission modes is simulated as a function of SNR using MIMO channel data extracted from our 3D ray tracer (see Section 4). The predicted channel data is combined with appropriately oriented complex polarimetric antenna patterns for the BS and each UE location.

Three data modulation schemes are supported in the LTE-A system (QPSK, 16-QAM, and 64-QAM) along with four different coding rates. The MCS modes considered in our simulator assume $2 \times 2 \mathrm{MIMO}$ and are listed in Table 2. The achievable throughputs at the PHY layer can be calculated from the error-free data rate and the residual PER. An approximation for throughput is given by throughput $=(1-\mathrm{PER}) \times$ $R_{b}$, where $R_{b}$ is the peak error-free transmission rate and PER is the residual packet error rate for a specific MCS mode.

The transmission date rate is defined as $R_{b}=$ $N_{\mathrm{SS}}\left(N_{d} r_{c} b N_{s}\right) / t_{\text {slot }}$, where $N_{\mathrm{SS}}$ denotes the number of 


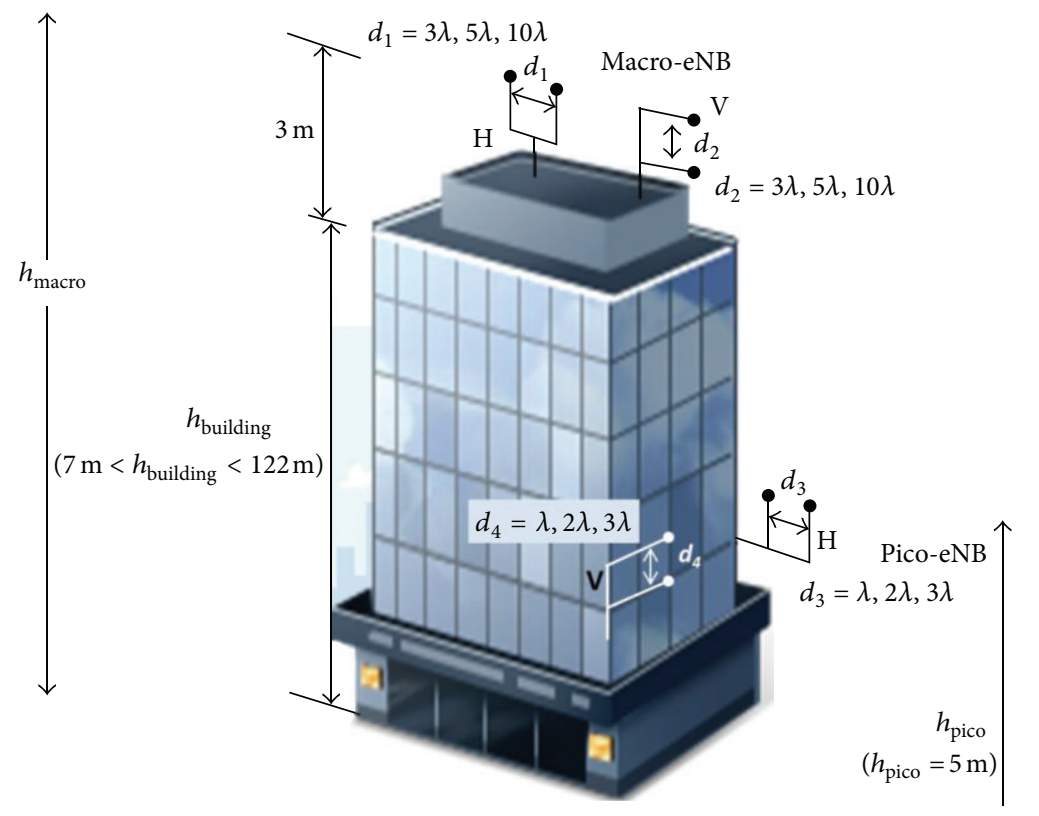

(a) BS
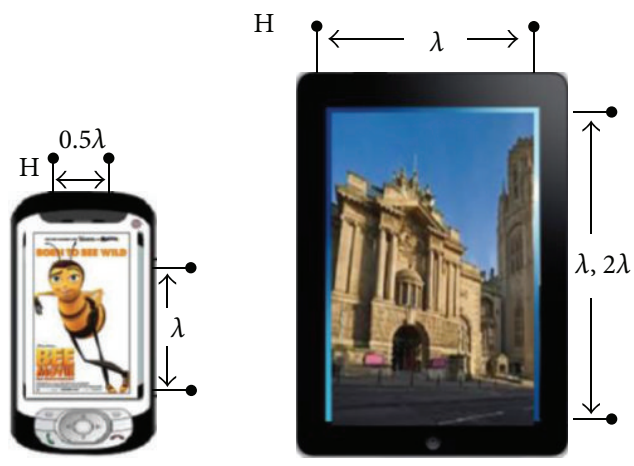

(b) Handset* portrait (c) Tablet* portrait

FIGURE 3: Illustrative view of antenna spacings and configurations at the BS and UE ( ${ }^{*}$ in practice the UE antennas are embedded within the handset casing).

spatial streams, $N_{d}$ is number of data subcarriers, $r_{c}$ is the coding rate, $b$ is the number of coded bits per subcarrier, $N_{s}$ is the number of OFDMA symbols per time slot, and $t_{\text {slot }}$ is the duration of a time slot. Based on Table 2 different MIMO modulation and coding schemes are chosen to match the SNR and spatial correlation properties of the channel.

In order to perform link-level analysis in an efficient and scalable manner, a PHY abstraction technique is required. In this paper, the RBIR abstraction technique is used to determine the optimal MCS mode. RBIR abstraction has been used by Kong et al. in $[15,16]$ and is fully described by Halls et al. in [17]. As described in Figure 1, the mode with the highest throughput (assuming PER $<10 \%$ ) is selected for each UE location $[18,19]$. The selection is optimum since it relies on simulating the throughput for all MCS modes for all UE locations.

Figure 2(a) shows the PER versus mean SNR from the full bit level LTE-A simulator in an ideal AWGN channel. This result was verified using the data reported in [20]. This data is used for link-to-system mapping in the RBIR abstraction engine. As shown in Figure 2(b), the abstraction approach has been thoroughly validated against our LTE-A link-level simulator in order to validate its accuracy.

In this investigation, the impact of MIMO antenna array configuration in an interference-free urban scenario is considered. As illustrated in Figure 3, both horizontal and vertical array configurations for various antenna spacings at the base station and handset are considered. The macrocell and picocell BSs were modelled to employ two horizontally and two vertically spaced antennas. Meanwhile, the UE was modelled with a maximum of two horizontally spaced antennas (to exploit the higher angle spread in the azimuth plane).
For each BS-UE link, a set of 1000 uncorrelated channel snap-shots is generated. Based on this specific channel data and antenna structure, the resulting PER and throughput are calculated. A noise floor of $-99.4 \mathrm{dBm}$ is assumed at the mobile receiver [19]. The terms handset or user equipment (UE), base station (BS) and eNode B (eNB) or macro eNode $\mathrm{B}$ (macro-eNB), and picobase station or pico eNode B (pico$\mathrm{eNB}$ ) are used interchangeably in this paper.

\section{Channel Modelling and Antennas}

The radiation patterns of a macrocell BS antenna (6-element array with patches in slant polarisation, downtilted by $10^{\circ}$ ), a picocell $\mathrm{BS}$ antenna (patch in slant polarisation), and a UE antenna (a common mobile phone) were measured in our anechoic chamber. All patterns are recorded in $3 \mathrm{D}$ and include full power, phase, and polarisation information. The 3D patterns provide insight into the behavior of the antenna systems that is simply not possible from single planes (e.g., $x-y, x-z$, and $y-z$ ). Figure 4 shows the antennas and their total power radiation patterns. Table 3 summarises the radiation pattern statistics. It should be noted that all the antennas in this study were assumed to be $100 \%$ efficient; thus their gain is equal to their directivity. Both ends of the link were modelled to employ two antennas of the same type separated by different distances in either the horizontal or vertical plane, resulting in different $2 \times 2$ MIMO system configurations. The propagation channel between each BS and UE was modelled via the spatial convolution of the polarimetric antenna patterns with the spatial and temporal multipath ray components from our $3 \mathrm{D}$ outdoor ray tracer [21-24]. 

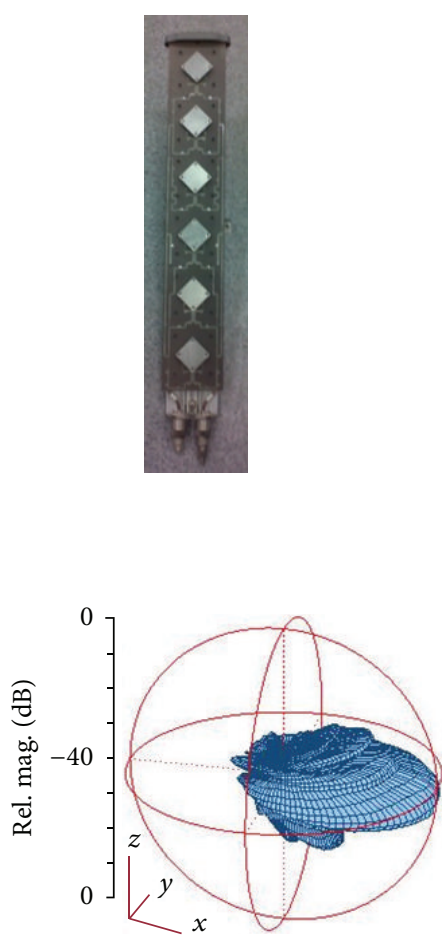

(a)

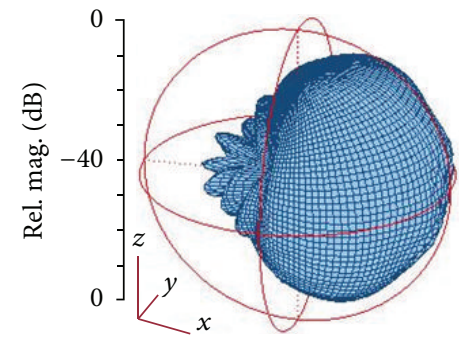

(b)
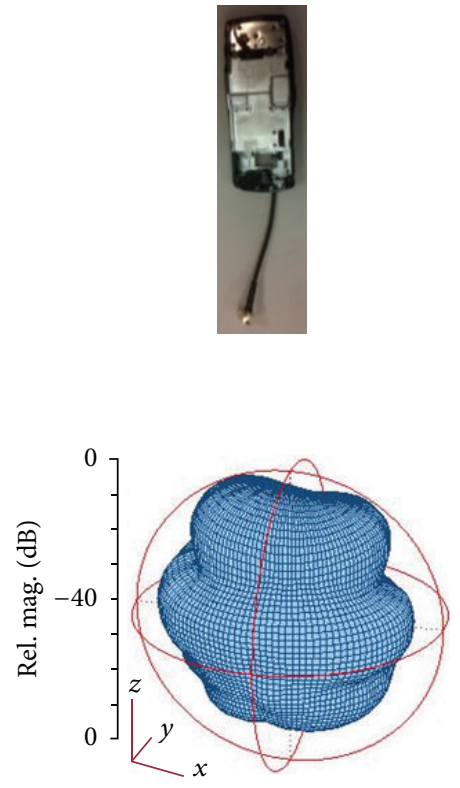

(c)

FIgURE 4: Measured antennas (top) and total power radiation patterns (bottom). (a) Macro-BS. (b) Pico-BS. (c) UE handset.

TABLE 3: Radiation pattern statistics.

\begin{tabular}{|c|c|c|c|c|}
\hline & \multicolumn{2}{|c|}{ Power in polarisation (\%) } & \multicolumn{2}{|c|}{ Maximum directivity (dBi) } \\
\hline & Vertical & Horizontal & Vertical & Horizontal \\
\hline Macro-BS & 47 & 53 & 12.5 & 13.8 \\
\hline Pico-BS & 40 & 60 & 7.0 & 5.9 \\
\hline UE handset & 43 & 56 & 6.8 & 6.3 \\
\hline
\end{tabular}

The ray-tracing engine identifies all possible ray paths between the base station and the mobile receiver and was used previously to generate the models reported in $[13,14$, 25]. The geographic database includes terrain, buildings, and foliage. The ray-tracing model has been validated for cellular (and picocellular) applications, where the base station was located above (and well below) the rooftop level at frequencies from $450 \mathrm{MHz}$ to $6 \mathrm{GHz}$, with a Root-meansquare-error (RMSE) of approximately $7 \mathrm{~dB}$ [21-24]. The urban environment database of a $17.6 \mathrm{~km}^{2}$ area in the centre of Bristol, UK, is investigated at a carrier frequency of $2.6 \mathrm{GHz}$.

For the macrocell study, 23 BSs were placed on rooftop locations (rooftop heights varied from $7 \mathrm{~m}$ to $122 \mathrm{~m}$ as shown in Figure 1). Each BS was modelled to cover a 3-sector cell, with a cell radius of $1 \mathrm{~km} .300$ UEs were randomly scattered in each sector within the BS antenna $3 \mathrm{~dB}$ beam width. The BS transmit power was $43 \mathrm{dBm}$, the BS antenna height was $3 \mathrm{~m}$ above rooftop level, and the UE antenna height was $1.5 \mathrm{~m}$ above ground level. A minimum distance of $50 \mathrm{~m}$ was assumed between the BSs and the UEs. For the picocell study, 600 BSs were randomly scattered at a height of $5 \mathrm{~m}$ above ground level. Each pico-BS was mounted on the external wall of a building and covered a 1-sector cell with a radius of $150 \mathrm{~m}$. 50 random UEs were considered per BS at a height of $1.5 \mathrm{~m}$ above ground level and within the BS antenna $3 \mathrm{~dB}$ beamwidth.

A pico-BS transmit power of $30 \mathrm{dBm}$ and a minimum BSUE separation distance of $5 \mathrm{~m}$ were assumed. Figures 5(a) and 5(b) show macrocell and selection of picocell examples. Figures 5(c) and 5(d) show examples of a macrocell and picocell coverage maps based on the total average received power at the user terminal. Base stations are denoted by red circles, while white crosses represent UE locations. A detailed statistical analysis of the propagation parameters can be found in $[25,26]$.

In order to compute a statistically valid set of wideband channel matrices suitable for Orthogonal Frequency Division Multiplexing (OFDM) modelling, the procedure reported in [27] was followed. Point-source ray tracing was conducted from each BS to each UE location. This provided information on the amplitude, phase, time delay, Angle of Departure (AoD), and Angle of Arrival (AoA) of each multipath component (MPC). The complex gain of each MPC was adjusted according to each transmitting/receiving antenna electric 


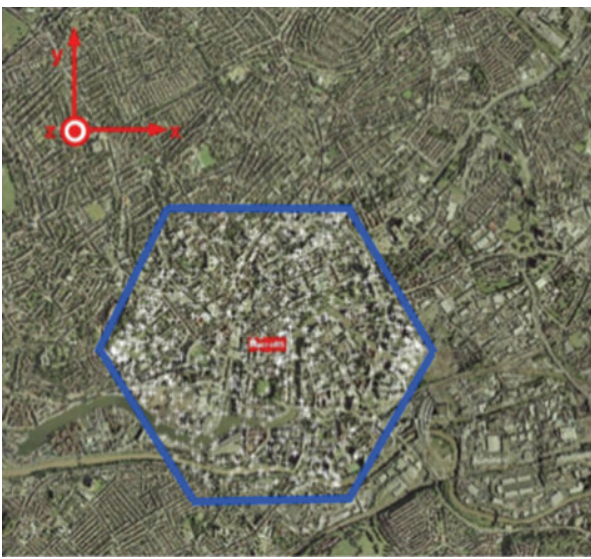

(a) Macrocell

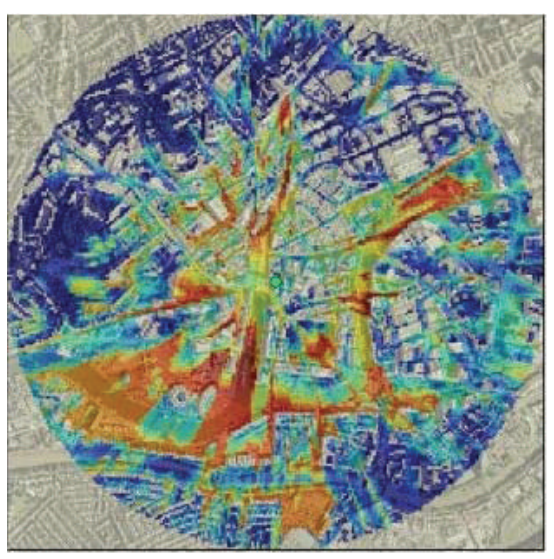

(c) Macrocell

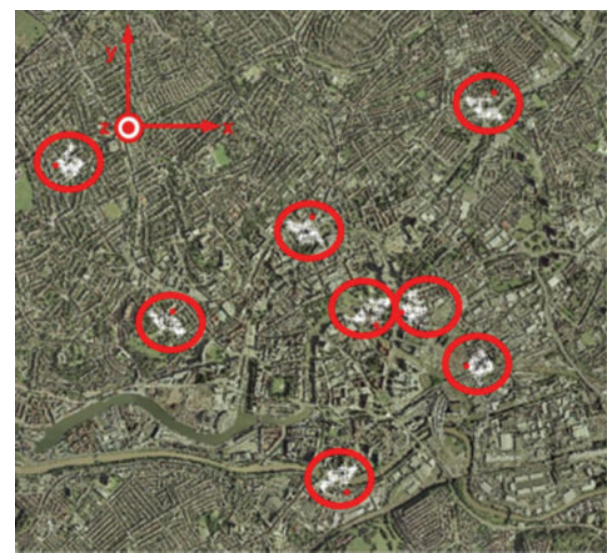

(b) Picocell
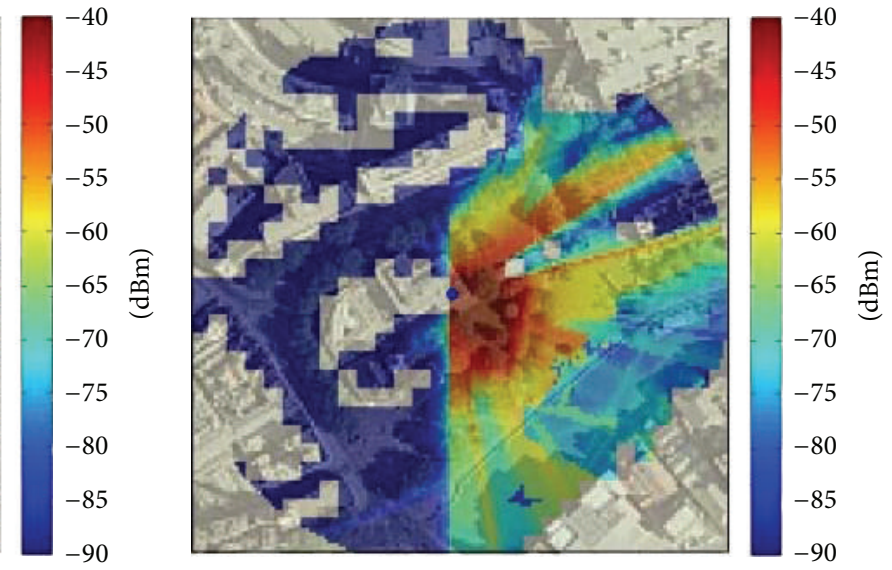

(d) Picocell

FigURE 5: Examples of HetNet scenario (a) and (b) and the coverage map for (c) macrocell and (d) picocell.

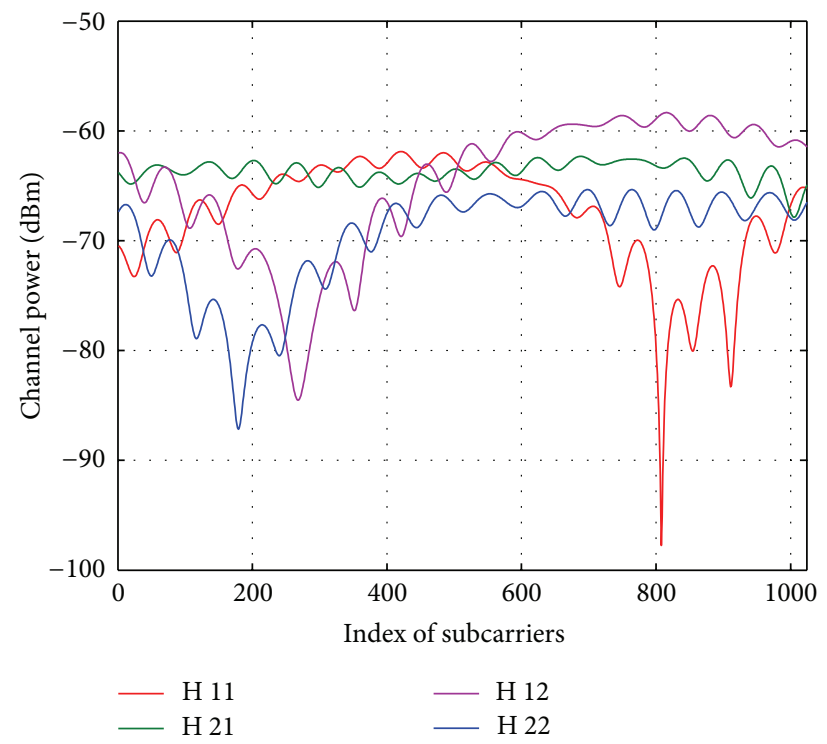

FIGURE 6: Instantaneous frequency power profile example for a $2 \times 2$ picocell link. 

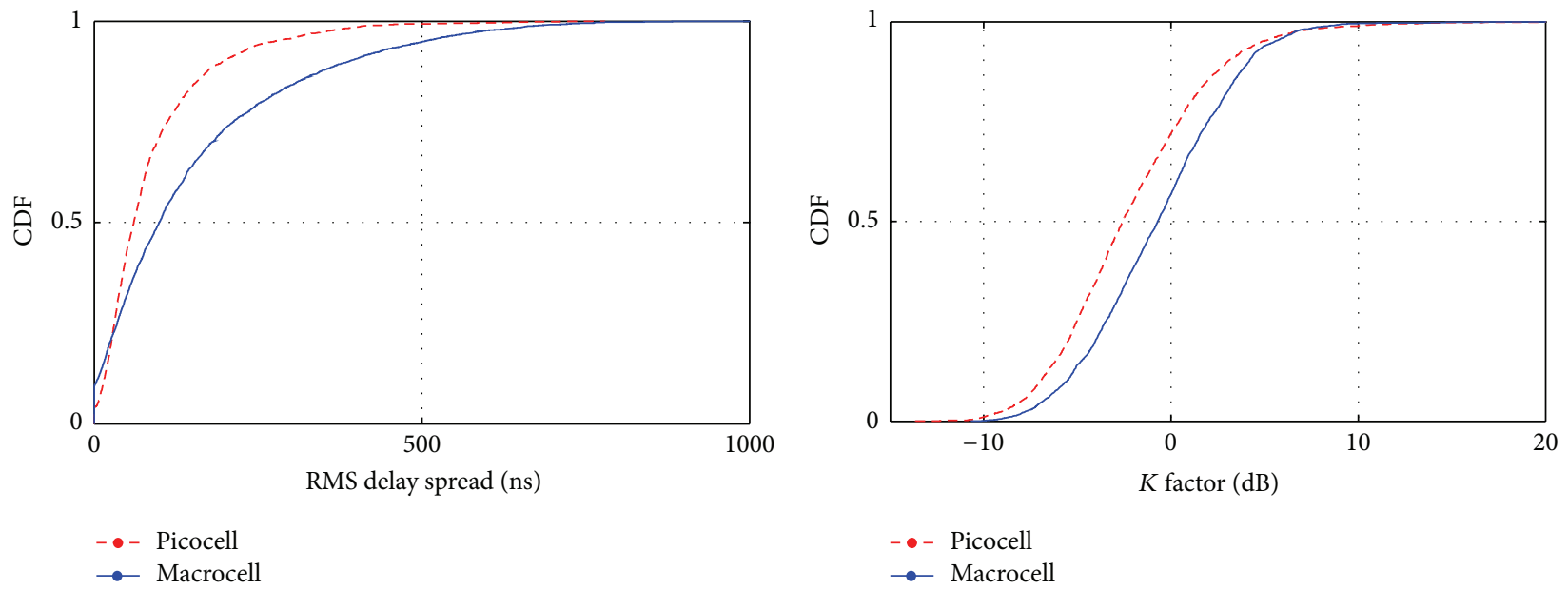

(a)

(b)
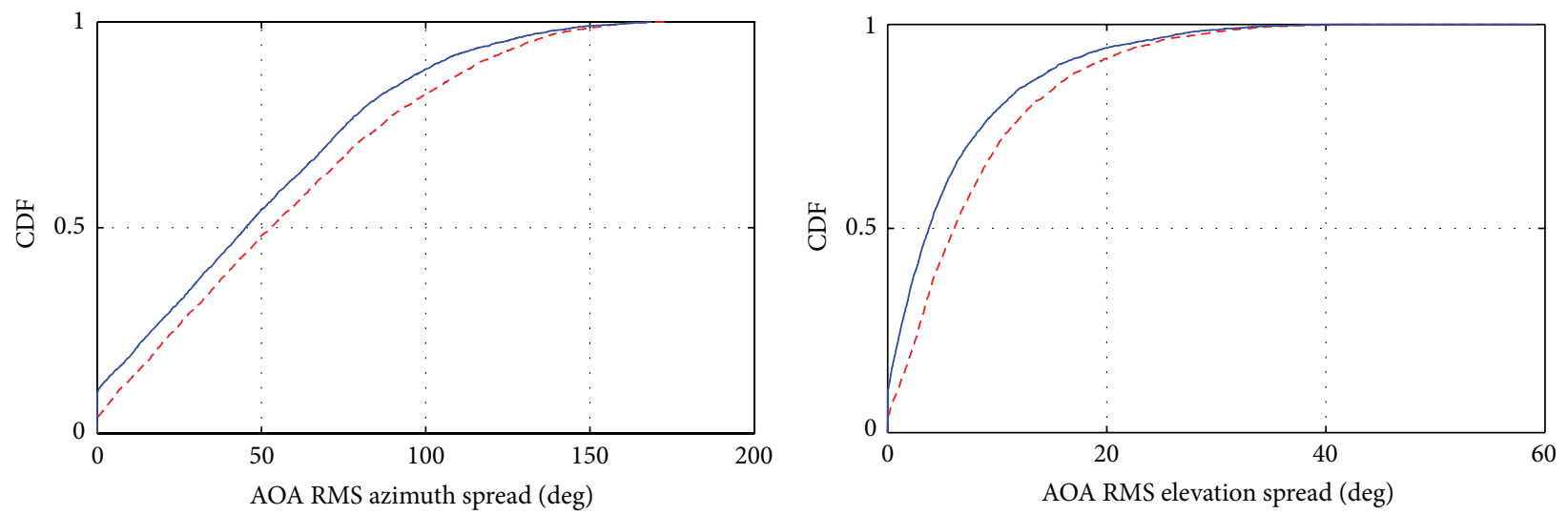

- - Picocell

- Macrocell

- - Picocell

$\rightarrow$ Macrocell

(c)

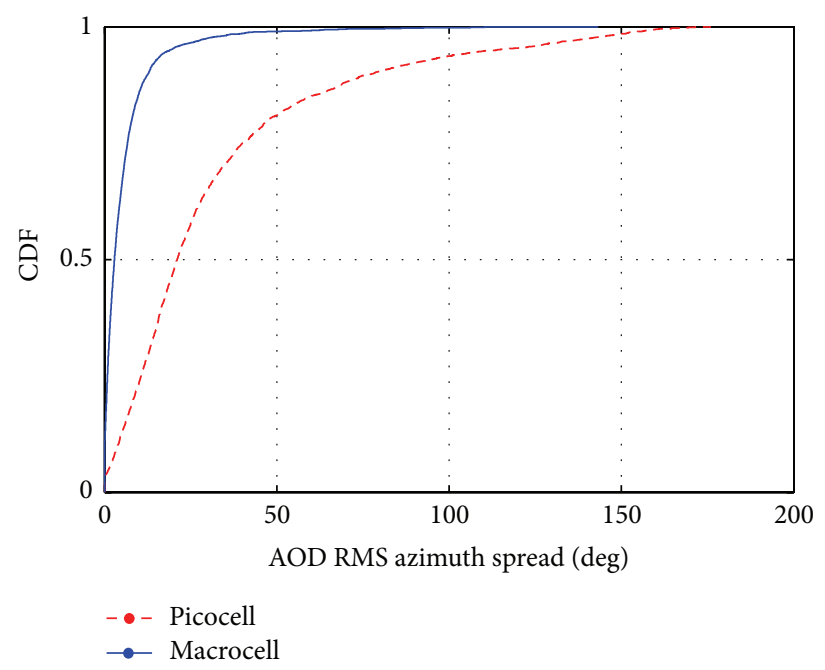

(e)

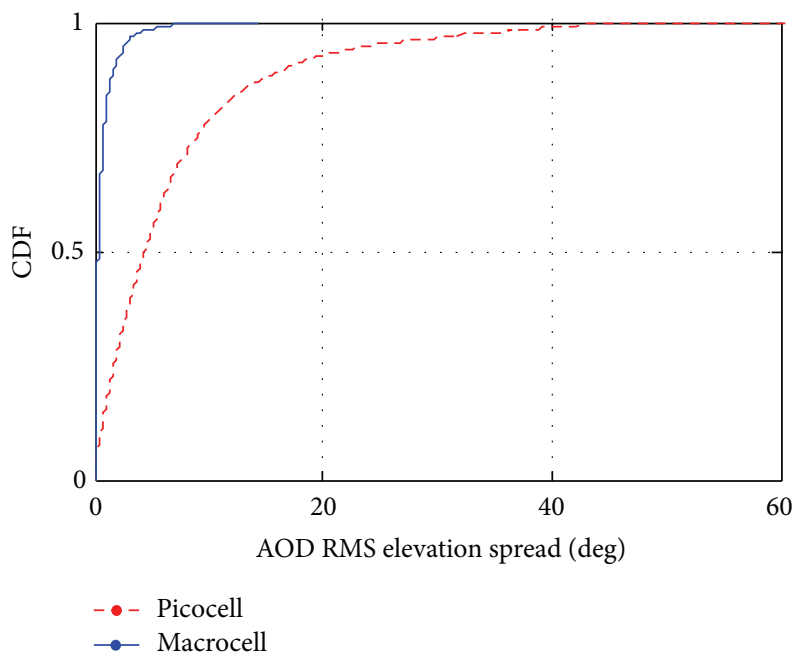

(f)

FIGURE 7: CDFs of macrocell and picocell propagation characteristics. 


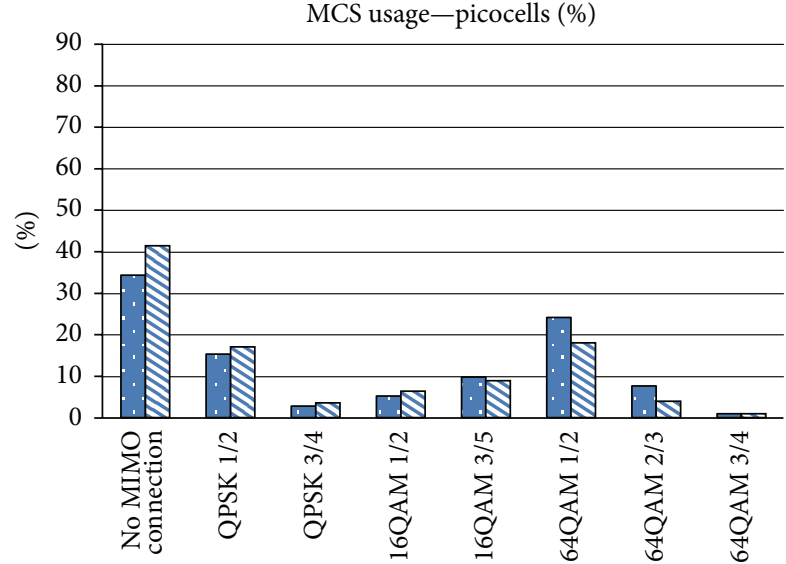

Modulation and coding schemes

(a) $3 \lambda$ eNB antenna spacing-Picocells

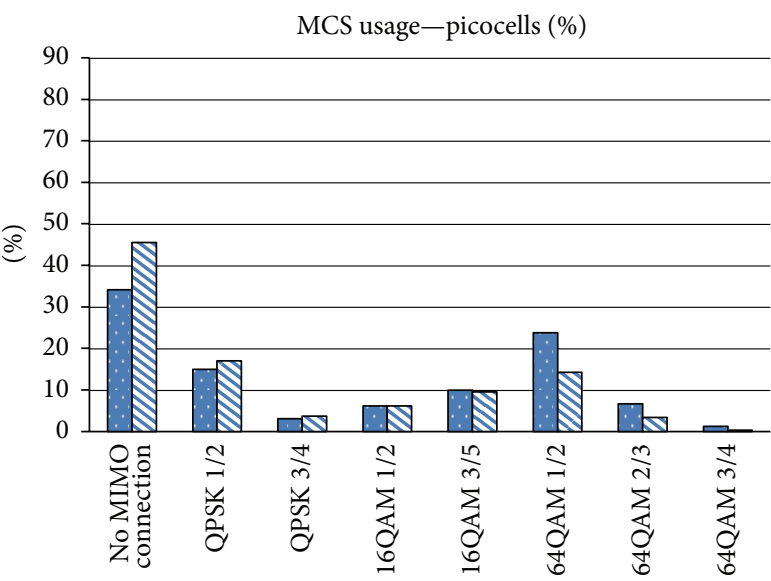

Modulation and coding schemes

Horizonta

$\mathbb{N}$ Vertical

(c) $2 \lambda$ eNB antenna spacing

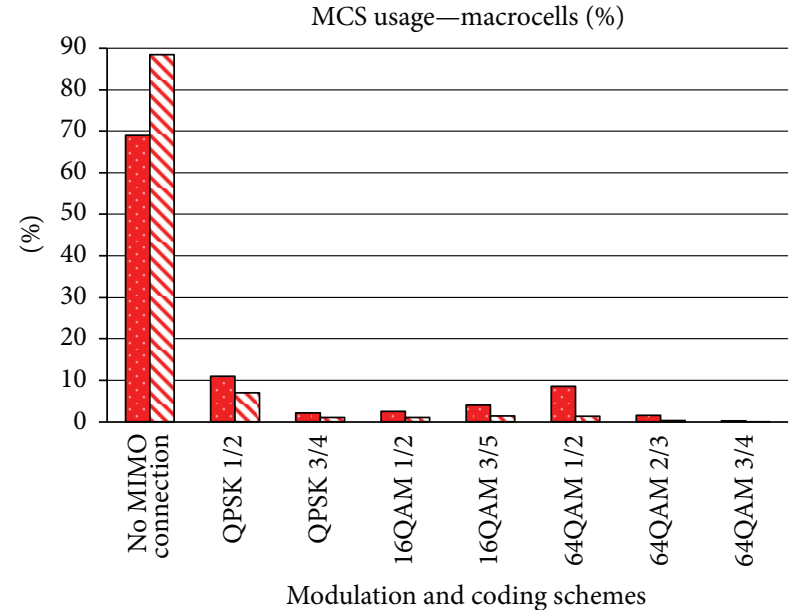

(b) $3 \lambda$ eNB antenna spacing-Macrocells

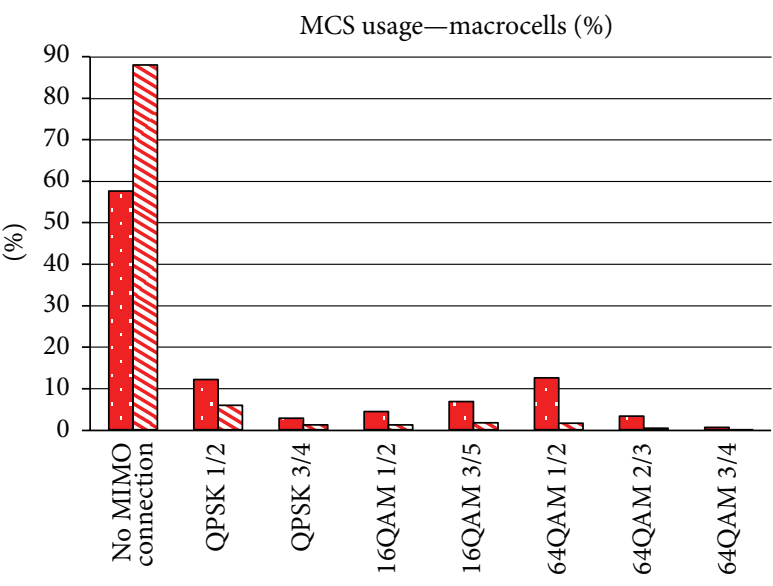

Modulation and coding schemes

Horizontal

$\mathbb{8}$ Vertical

(d) $5 \lambda$ eNB antenna spacing

FIgURE 8: Picocell and macrocell MCS selection of horizontal and vertical eNB configurations at $15 \mathrm{~dB}$ receive SNR (NLoS links only).

field pattern response for the corresponding AoD/AoA and polarisation. The double-directional time-invariant channel impulse response $h_{m n}$ for the $m$ th transmitting and $n$th receiving antenna links is given by [28]

$$
\begin{aligned}
h_{m n_{L}} & \left(\tau, \Omega_{\mathrm{AoD}}, \Omega_{\mathrm{AoA}}\right) \\
= & \sum_{l=1}^{L} h_{m n l}\left(\tau, \Omega_{\mathrm{AoD}}, \Omega_{\mathrm{AoA}}\right) \\
= & \sum_{l=1}^{L} E_{m n l} \delta\left(\tau-\tau_{l}\right) \delta\left(\Omega_{\mathrm{AoD}}-\Omega_{\mathrm{AoD}, l}\right) \delta\left(\Omega_{\mathrm{AoA}}-\Omega_{\mathrm{AoA}, l}\right),
\end{aligned}
$$

where

$$
E_{m n l}=\left[\begin{array}{l}
E_{T x, m}^{V} \\
E_{T x, m}^{H}
\end{array}\right]^{T}\left[\begin{array}{ll}
a_{l}^{V V} e^{j \varphi_{l}^{V V}} & a_{l}^{V H} e^{j \varphi_{l}^{V H}} \\
a_{l}^{H V} e^{j \varphi_{l}^{H V}} & a_{l}^{H H} e^{j \varphi_{l}^{H H}}
\end{array}\right]\left[\begin{array}{c}
E_{R x, n}^{V} \\
E_{R x, n}^{H}
\end{array}\right],
$$

where $\delta(\cdot)$ represents the Dirac delta function, $\tau$ is the time of flight, $\Omega_{\mathrm{AoD}} / \Omega_{\mathrm{AoA}}$ represents the departure/arrival angle, and $L$ is the total number of MPCs. The $l$ th MPC between the $m$ th transmitting and the $n$th BS antennas has a double-directional time-invariant channel impulse response $h_{m n l}$, a complex amplitude $a_{l}^{X Y} e^{j \varphi_{l}^{X Y}}($ a $2 \times 2$ matrix for all four polarisation combinations), a time of flight $\tau_{l}$, and departure/arrival solid angles $\Omega_{\mathrm{AoD}, l} / \Omega_{\mathrm{AoA}, l}$, and $E_{T x, m}^{V / H} / E_{R x, n}^{V / H}$ represents the vertical/horizontal polarisation component of the $m$ th transmitting $/ n$th BS receiving antenna electric field radiation patterns. Time binning was then applied with a bin resolution of $32.55 \mathrm{~ns}$. The wideband channel frequency response $G_{m n}(f)=\left[g_{m n 1}, g_{m n 2}, \ldots, g_{m n N}\right]$, where $g_{m n k}$ represents the frequency-domain channel for the $m$ thtransmitting antenna, the $n$ th-receiving antenna, and the $k$ th subcarrier (the total number of subcarriers is $N$ ), was computed using a 2048-point Discrete Fourier Transform (DFT) as shown in

$$
G_{m n}(f)=F\left\{h_{m n}\right\},
$$

where $F\{\cdot\}$ represents the DFT. 


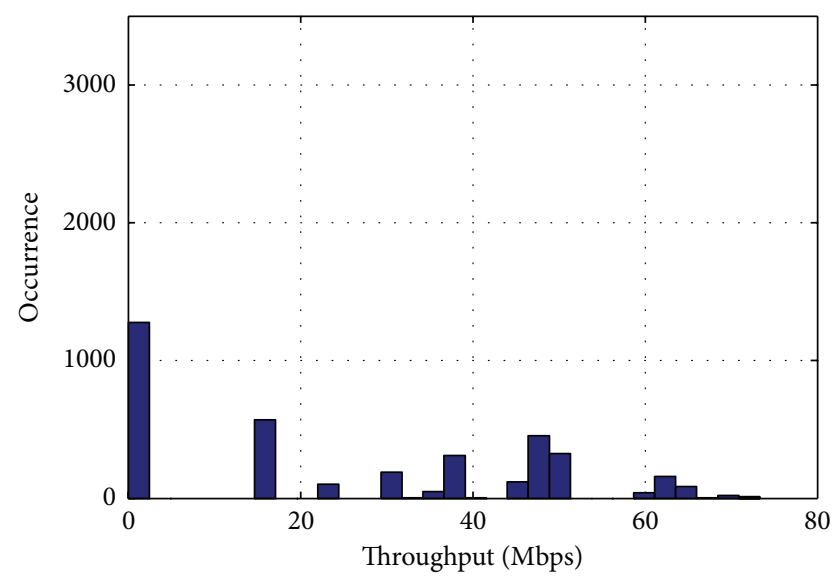

$\square$ Pico horizontal $3 \lambda$

(a)

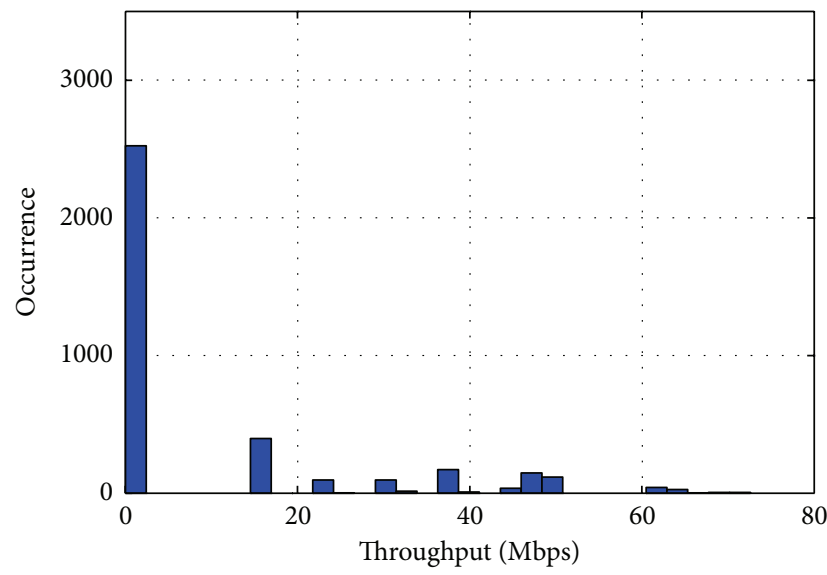

Macro horizontal $3 \lambda$

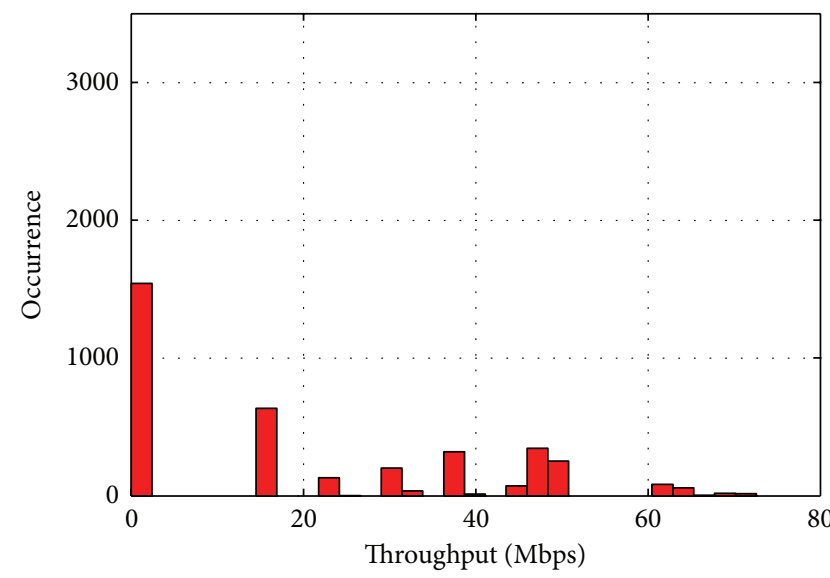

Pico vertical $3 \lambda$

(b)

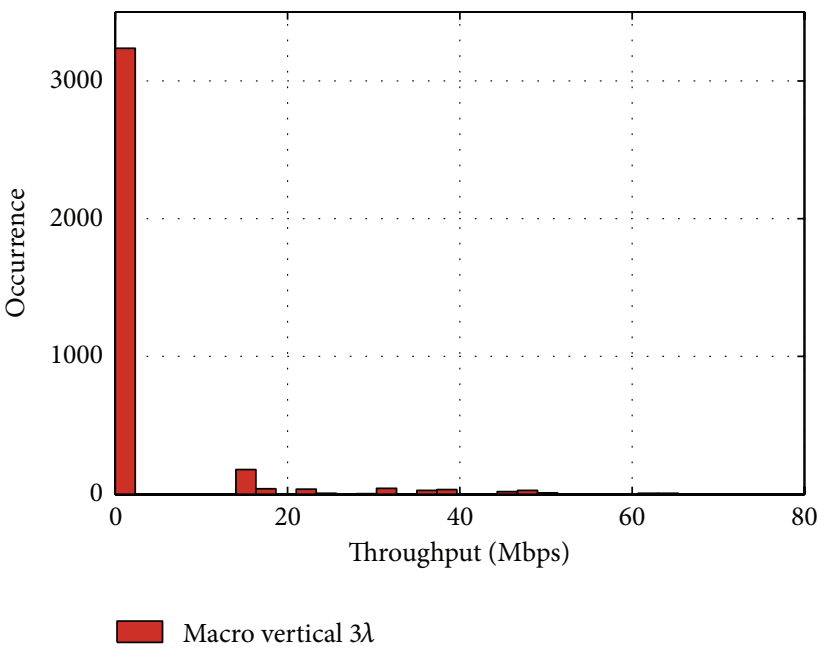

(d)

FIGURE 9: Throughput occurrence statistics for vertical and horizontal eNB antenna array configurations (NLoS links only) with BS antenna separation of $3 \lambda$ (total number of occurrences is 3,729 for picocells and 3,681 for macrocells).

An example of a set of instantaneous wideband channel matrices can be seen in Figure 6 for a $2 \times 2$ picocell link. To compute the average channel performance for a given link, this procedure was repeated for 1000 independent channel realizations, applying a uniformly distributed $[0,2 \pi)$ random phase to each MPC.

The previous process resulted in 7,283 pico-UE links and 4,076 macro-eNB-UE links. The process is summarised by the following 8 steps:

(1) associate UEs to BSs;

(2) ray trace each UE-BS link to determine relevant multipath components (in 3D space);

(3) compute a set of $1000 \mathrm{H}$-matrices for each UE-BS link based on isotropic antennas;

(4) spatially and polarimetrically convolve the measured $\mathrm{BS}$ and UE antenna patterns with the 3D channel data;
(5) compute a second set of $1000 \mathrm{H}$-matrices for each BSUE link;

(6) perform RBIR abstraction to determine the LTEA link performance based on the BS-UE SNR and scenario specific MIMO channel $H$-matrices;

(7) determine PER and link throughput for all MCS and MIMO modes;

(8) select the MCS mode that maximises link throughput given PER $<10 \%$.

Repeat the process for all BS-UE links.

The macrocell and picocell propagation statistics are presented in Figure 7 in terms of CDFs for K-factor, RMS delay spread, and RMS azimuth and elevation spreads (for the base station and user terminal). For the macrocells the values of the RMS azimuth spread at the base station (AoD) far exceed the values of the RMS elevation spread. It can also be 


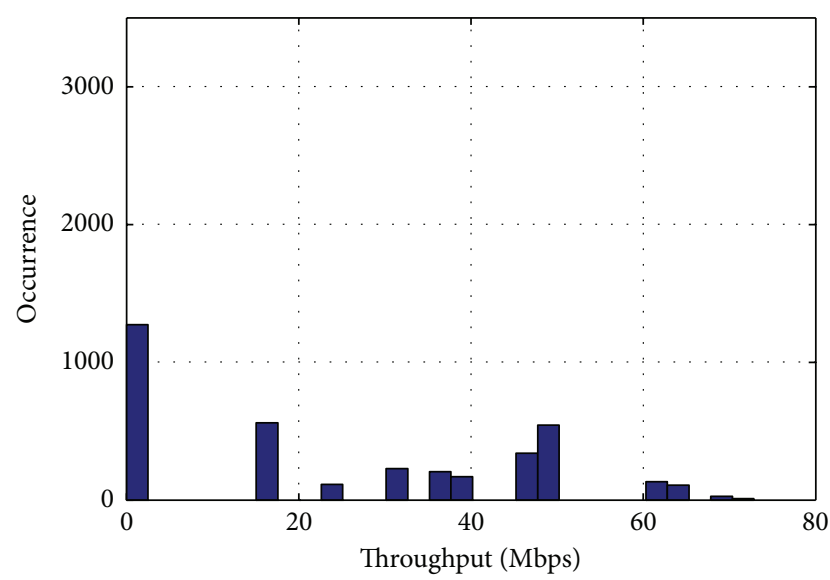

Pico horizontal $2 \lambda$

(a)

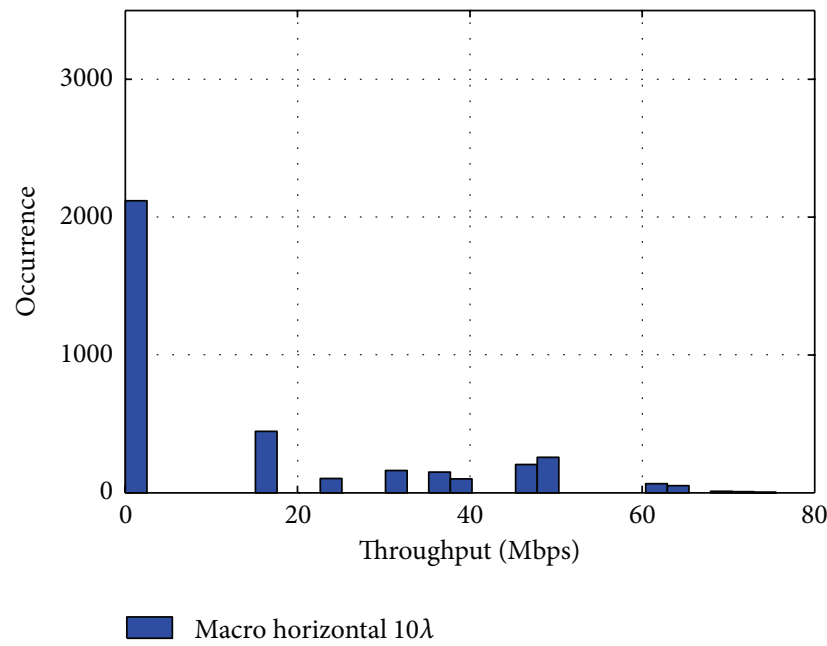

(c)

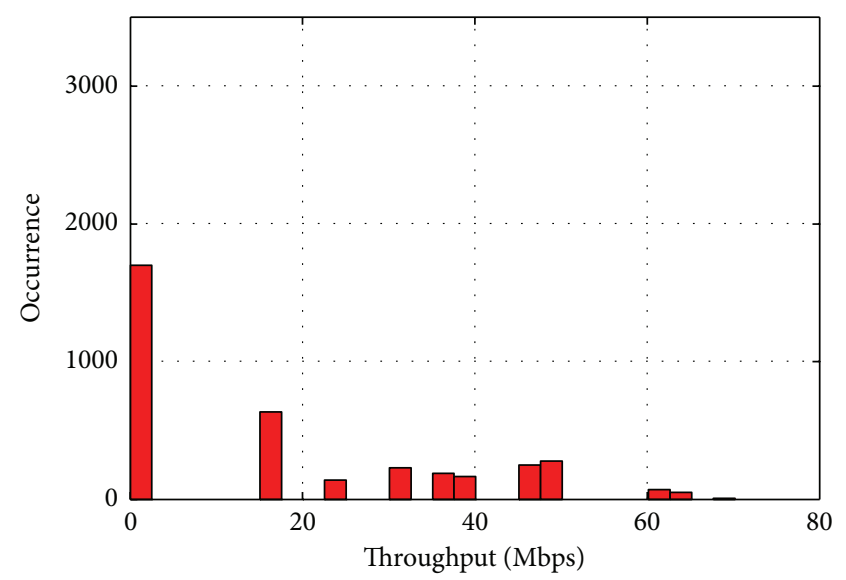

(b)

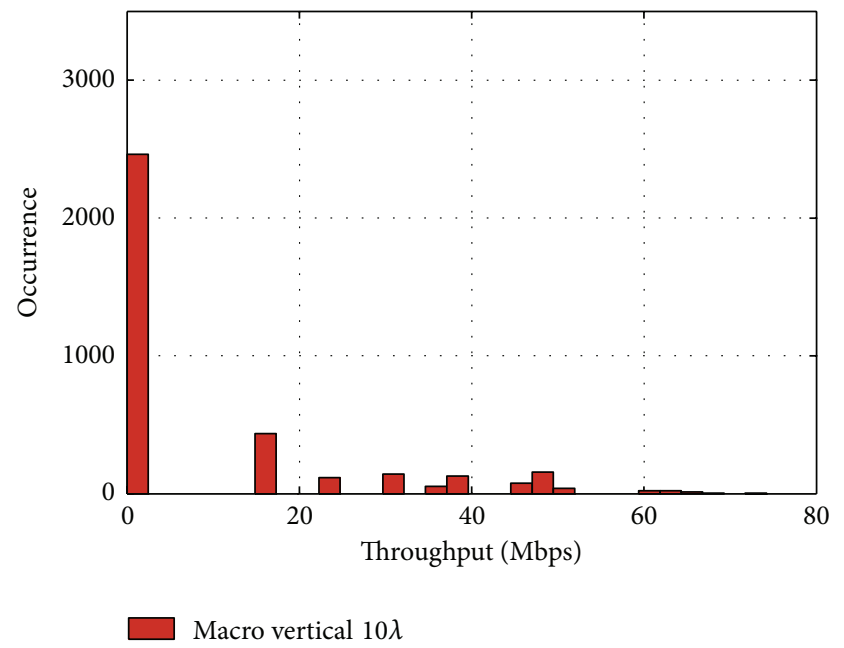

(d)

FIGURE 10: Throughput occurrence statistics for vertical and horizontal eNB antenna array configurations (NLoS links only) with various BS antenna separations (total number of occurrences is 3,729 for picocells and 3,681 for macrocells).

seen that for picocellular base stations there is a considerable increase (relative to the macrocells) in both the RMS azimuth and RMS elevation spreads. Figure 7 also shows that the RMS delay spreads are statistically higher in the macrocells (since they illuminate a larger volume of the city), as are the values of K-factor (higher mounted macrocells are more likely to generate a dominant multipath component).

\section{PHY Layer Performance Evaluation}

This section presents results from our LTE-A PHY layer simulator [11]. In particular the performance of the $2 \times 2 \mathrm{DL}$ SM MIMO OFDMA physical layer is investigated for NLoS UE terminals connected to horizontal and vertical MIMO arrays at the eNodeB. The $3 \mathrm{D}$ ray-tracing model introduced in Section 4 is used to compute the MIMO channel response matrices for each user. Results are presented in terms of the PER, mean throughput, and eigenvalue spread. Link selection is performed for each user, and a UE is assumed to be in outage if none of the MIMO-enabled MCS modes are able to meet the $10 \%$ PER threshold.

5.1. Throughput Performance Analysis. Figure 8 shows the usage (number of occurrences) of each modulation and coding scheme (assuming an SNR of $15 \mathrm{~dB}$ ) from QPSK 1/2 (lowest data rate) to 64QAM 3/4 (highest data rate) when the eNB uses either a horizontal or vertical array. Antenna spacings of $2 \lambda$ and $3 \lambda$ are explored for picocells and $3 \lambda$ and $5 \lambda$ for macrocells. The graphs show that the higher-order MIMO modes are more likely to be selected in the picocellular environment. There are significantly less outage cases (denoted by "No MIMO Connection") for the picocellular scenarios compared to the macrocellular scenarios.

Furthermore, for picocellular eNodeBs, the use of vertical arrays is now seen as a viable option. In the macrocells the usage of MIMO modes is seriously compromised by low 


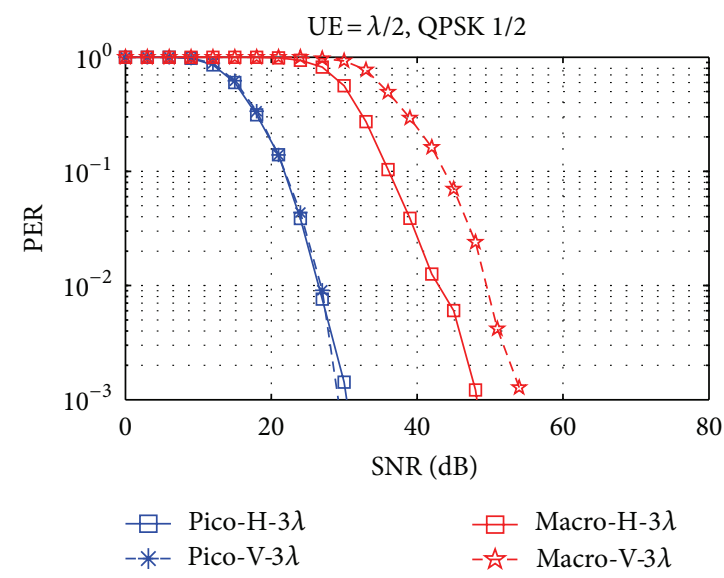

(a) $3 \lambda$ eNB antenna separation

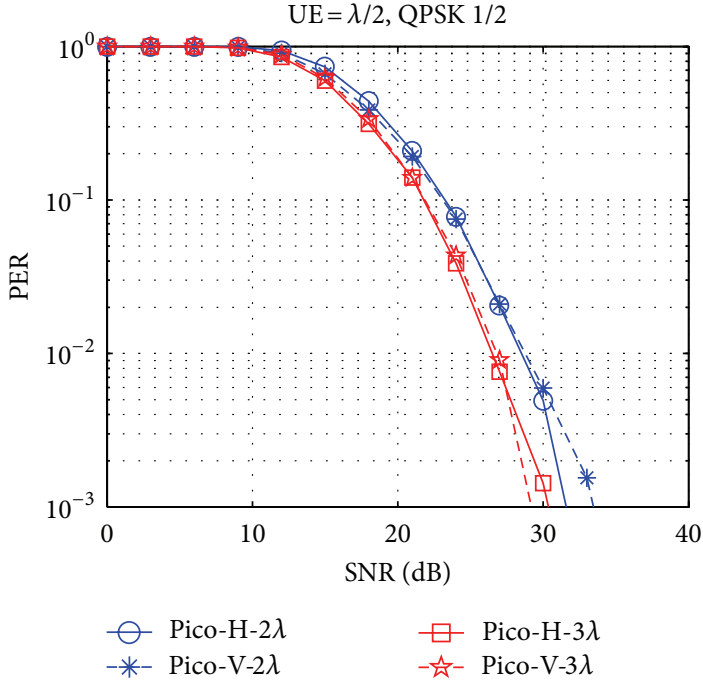

(b) Picocell

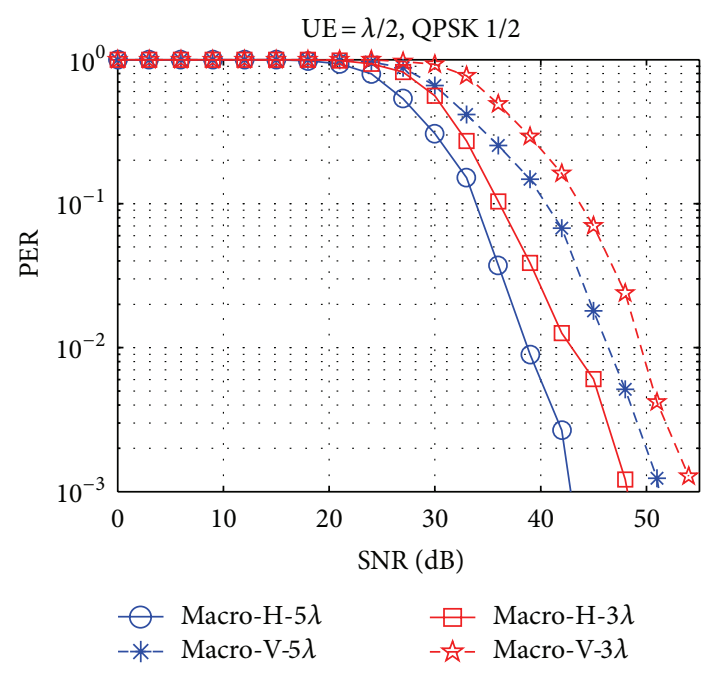

(c) macrocell

FIGURE 11: PER performance as a function of SNR for horizontal and vertical eNB antenna array configurations at different eNB antenna separations.

angular spread in both the azimuth and elevation planes. In addition, the throughput occurrence rates for both the vertical and horizontal eNB antenna configurations are shown in Figures 9 and 10. It is interesting to note that the picocells offer significantly higher data rates compared to the macrocells. In both scenarios increasing the eNB antenna spacing has little overall effect in the picocells; however, this has a significant impact in the macrocells.

5.2. Packet Error Rate Performance Analysis. The PER performance for QPSK is compared for NLoS locations in a picoand macrocell for different antenna spacings in Figure 11. The UE antenna separation in this analysis is maintained at $\lambda / 2$. The PER performance is also shown for horizontal and vertical eNB antenna configurations. Perfect channel estimation is assumed along with a packet size of 54 bytes. All results are averaged over 1000 channel realisations unless otherwise stated. From Figure 11(a) it can be seen that that macroperformance using a vertical eNB array is very poor (around $23 \mathrm{~dB}$ worse than the equivalent picocellular channel). Figure 11(b) confirms that the vertical and horizontal eNB arrays offer similar performance in the picocell. However, as shown in Figure 11(c), it is clear that the vertical eNB configuration for a macrocell results in significantly degraded performance (relative to a traditional horizontal configuration). In the picocell, the horizontal configuration offers a $0.3 \mathrm{~dB}$ gain over the vertical configuration. However, for the same PER target in the macrocell, the vertical eNB antenna configuration is $6.5 \mathrm{~dB}$ worse than the horizontal case. Figures 11 (b) and 11(c) present results for different $\mathrm{eNB}$ antenna separations. It can be seen that larger antenna separations improve the PER performance in both environments. As shown in Figure 11(b), the results for $2 \lambda$ spacing in the picocell are around $2 \mathrm{~dB}$ worse than the use of $3 \lambda$ spacings. Figure 11(c) shows the same scenario for the macrocell. The macrocell with $5 \lambda$ 


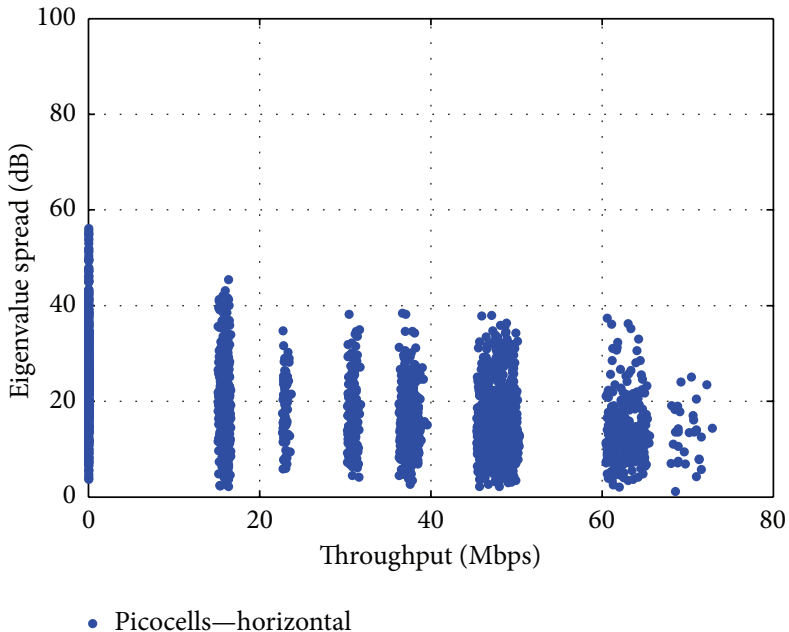

(a)

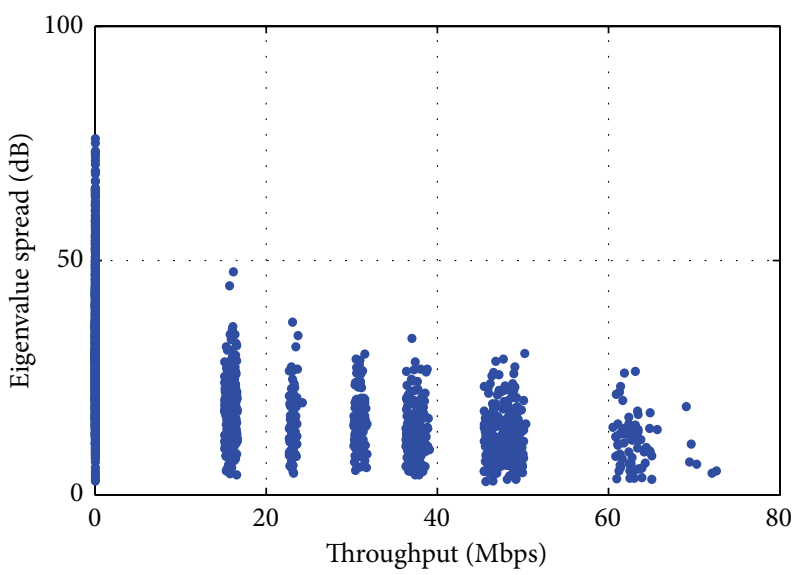

- Macrocells-horizontal

(c)

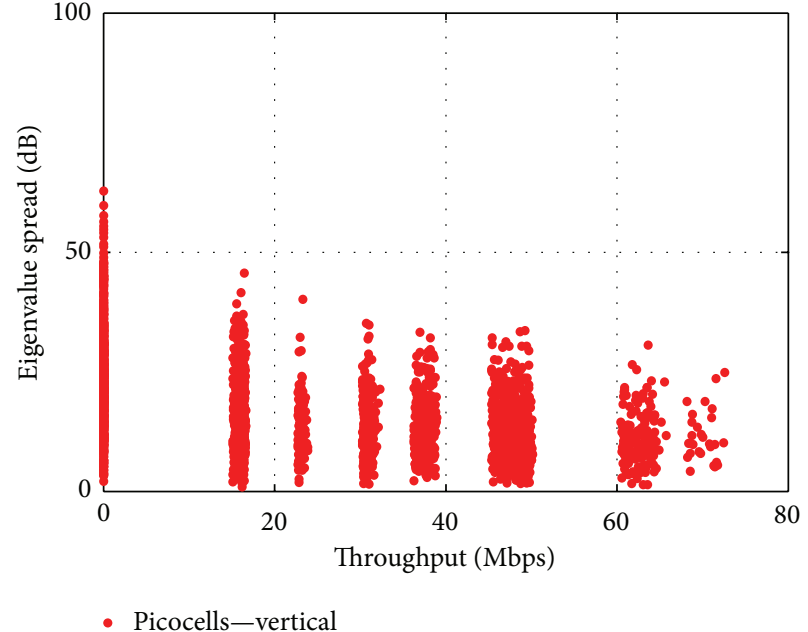

(b)

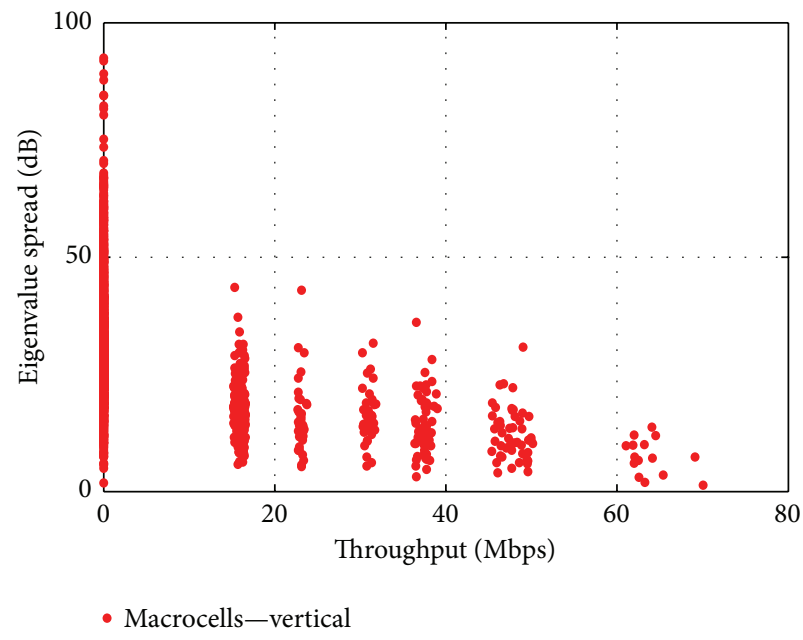

(d)

FIGURE 12: Scatter plot of throughput versus eigenvalue spread for picocells and macrocells with horizontal and vertical eNB antenna configurations and an antenna separation of $3 \lambda$ (NLoS links only).

eNB antenna spacing has an $8 \mathrm{~dB}$ gain over the $3 \lambda$ case for horizontal orientations and $3 \mathrm{~dB}$ for vertical configurations.

\subsection{Signal Correlation Performance Analysis. Figure 12 shows} the relationship between the throughput (in Mbps) and the eigenvalue spread of the MIMO channel correlation matrix at a fixed SNR of $15 \mathrm{~dB}$ for the picocell and macrocell scenarios, respectively. High eigenvalue spreads indicate high spatial correlation in the eNB-UE links. Spatial correlation is directly linked to angle spread at the base station and UE terminal. In the picocells, where the elevation angle spread at the eNB is much closer to the azimuth values, the performance of vertically spaced arrays is similar to this of horizontally spaced arrays. However, in the macrocell the throughput performance for vertical element spacings at the eNB is much worse than for traditional horizontal spacings. Overall, we see that the higher throughput modes in LTE-A require lower values of eigenvalue spread.
Figure 13 shows the probability of the eigenvalue spread being less than the abscissa for horizontal and vertical eNB antenna configurations for various eNB antenna separations. It can be seen that vertical configurations degrade the eigenvalue spread in macrocells far more than in picocells. In all cases horizontal eNB antennas achieve better levels of spatial decorrelation (i.e., lower eigenvalue spreads). This result is particularly noticeable for the macrocell base stations since the elevation spreads in this scenario are significantly lower than the azimuth values.

\section{Conclusions}

This paper has combined polarimetric base station and UE 3D antenna patterns with state-of-the-art ray tracing to analyse the performance of horizontal and vertical MIMO arrays in an urban $2.6 \mathrm{GHz}$ LTE-A network. Our propagation models were built on those used to guide the development of 


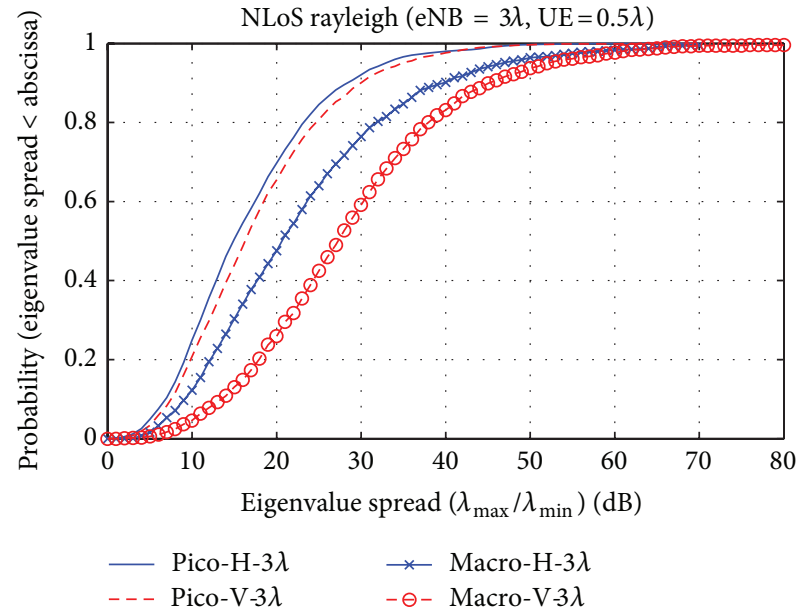

(a) $\mathrm{BS}$ antenna spacing $=3 \lambda$ and UE antenna spacing $=0.5 \lambda$

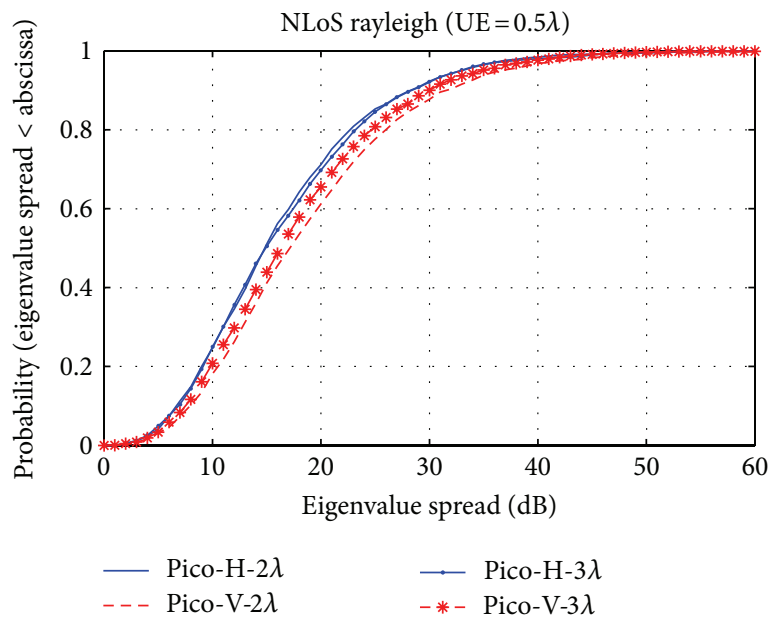

(b) BS antenna spacing $=2 \lambda, 3 \lambda$ and UE antenna spacing $=0.5 \lambda$

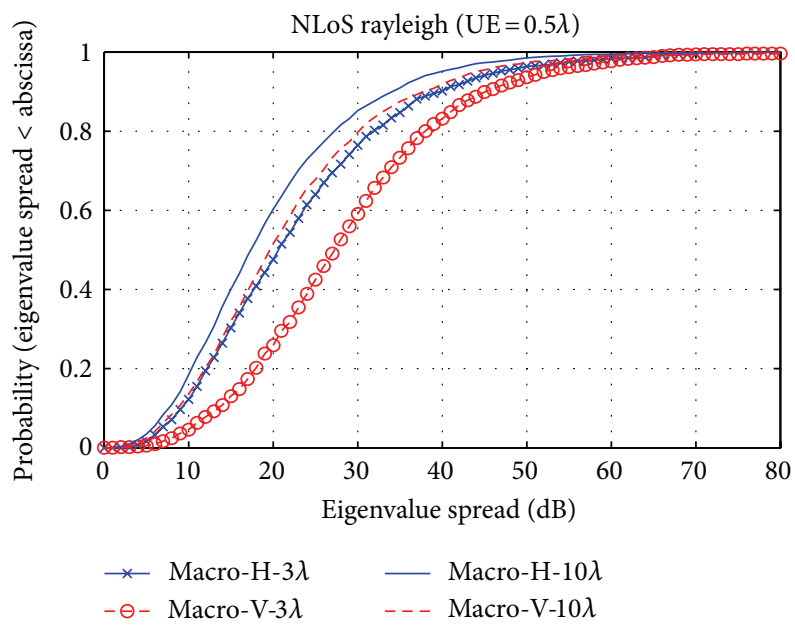

(c) BS antenna spacing $=3 \lambda, 10 \lambda$ and UE antenna spacing $=0.5 \lambda$

Figure 13: The CCDF of eigenvalue spread performance of picocells and macrocells in horizontal and vertical eNB antenna array configurations (NLoS links only).

3D channel model extensions in 3GPP. Results showed that for picocells the elevation angular spread at the base station was significantly increased relative to traditional microcells. A small increase was also observed here in the azimuth angle spread. Furthermore, our picocell studies demonstrated reduced values of RMS delay spread and Rician K-factor.

By combining the output of our detailed ray model with an LTE-A PHY layer simulator, it was possible to relate antenna and propagation statistics to PHY layer data rates and MIMO modulation and coding schemes. Results showed that vertical arrays at the eNodeB performed very poorly in macrocells but offered viable performance enhancement in picocells due to their increased levels of elevation angular spread.

Results showed that $3 \mathrm{D}$ angular statistics are necessary at the eNodeB and UE in order to adequately quantify the performance of LTE-A links. This paper has shown that picocells, which play a key capacity enhancing role in LTE HetNet configurations, are able to exploit vertical element spacings to deliver more compact antenna arrays at the base station.

\section{Acknowledgments}

Zuhanis Mansor would like to thank the Majlis Amanah Rakyat (MARA) and the Universiti Kuala Lumpur (UniKL) for her postgraduate scholarship and the Communication Systems and Networks Group, University of Bristol, for the provision of the simulation and laboratory facilities.

\section{References}

[1] “3GPP Release 10 V0.1.1, Overview of 3GPP” 2011.

[2] N. Prasad, H. Zhang, M. Jiang, G. Yue, and S. Rangarajan, "Resource allocation in 4G MIMO cellular uplink," in Proceedings of the 54th Annual IEEE Global Telecommunications Conference: Energizing Global Communications (GLOBECOM '11), pp. 1-5, December 2011. 
[3] S. Li, Q. Cui, C. Wang, and X. Tao, "Coordinated cell threshold user pairing criteria for uplink CoMP MU-MIMO," in Proceedings of the International Conference on Computer Science and Network Technology (ICCSNT '11), pp. 1740-1744, December 2011.

[4] J. Lee, J.-K. Han, and J. Zhang, "MIMO technologies in 3GPP LTE and LTE-advanced," Eurasip Journal on Wireless Communications and Networking, vol. 2009, no. 1, Article ID 302092, 2009.

[5] Cisco Visual Networking Index, Global Mobile Data Traffic Forecast Update, 2009-2014, Cisco Visual Networking Index, San Jose, Calif, USA, 2010.

[6] A. Khandekar, N. Bhushan, J. Tingfang, and V. Vanghi, "LTEadvanced: heterogeneous networks," in Proceedings of the 2010 European Wireless Conference (EW'10), pp. 978-982, April 2010.

[7] J. Hoadley and P. Maveddat, "Enabling small cell deployment with HetNet," IEEE Wireless Communications, vol. 19, no. 2, pp. 4-5, 2012.

[8] S. Landström, H. Murai, and A. Simonsson, "Deployment aspects of LTE pico nodes," in Proceedings of the IEEE International Conference on Communications Workshops (ICC '11), pp. $1-5$, June 2011.

[9] O. Stanze and A. Weber, "Heterogeneous networks with LTEadvanced technologies," Bell Labs Technical Journal, vol. 18, no. 1, pp. 41-58, 2013.

[10] K. Hiltunen, "Comparison of different network densification alternatives from the LTE uplink point of view," in Proceedings of the IEEE 22nd International Symposium on Personal, Indoor and Mobile Radio Communications (PIMRC '11), pp. 1601-1605, September 2011.

[11] Z. Mansor, E. Mellios, A. Nix, J. McGeehan, and G. Hilton, "Impact of antenna patterns and orientations in heterogeneous LTE-Advanced networks," in Proceedings of the 6th European Conference on Antennas and Propagation (EUCAP '12), pp. 1904-1908, 2012.

[12] T. Thomas, F. W. Vook, E. Visotsky et al., "3D extension of the 3GPP/ITU channel model," in Proceedings of the 77th IEEE Vehicular Technologies Conference (VTC-Spring), Dresden, Germany, May 2013.

[13] Text Proposal R1-130497, "3D Channel Modeling Issues and 3D Channel Model Proposal, 3GPP TSG-RAN WG1”.

[14] Text Proposal R1-130500, "Detailed 3D Channel Model, 3GPP TSG-RAN WG1".

[15] D. Kong, E. Mellios, D. Halls, A. Nix, and G. Hilton, “Throughput sensitivity to antenna pattern and orientation in $802.11 \mathrm{n}$ networks," in Proceedings of the IEEE 22nd International Symposium on Personal, Indoor and Mobile Radio Communications (PIMRC '11), pp. 809-813, September 2011.

[16] D. Kong, E. Mellios, D. Halls, A. Nix, and G. Hilton, "Closedloop antenna selection for wireless LANs with directional \& omni-directional elements," in Proceedings of the IEEE 74th Vehicular Technology Conference (VTC Fall '11), pp. 1-5, September 2011.

[17] D. Halls, A. Nix, and M. Beach, "System level evaluation of UL and DL interference in OFDMA mobile broadband networks," in Proceedings of the IEEE Wireless Communications and Networking Conference (WCNC '11), pp. 1271-1276, March 2011.

[18] F. Athley and M. N. Johansson, "Impact of electrical and mechanical antenna tilt on LTE downlink system performance," in Proceedings of the IEEE 71st Vehicular Technology Conference (VTC '10), pp. 1-5, May 2010.
[19] M. Rumney, LTE and the Evolution to $4 G$ Wireless, Agilent Technologies, Santa Clara, Calif, USA, 1st edition, 2009.

[20] L. Korowajczuk, LTE, WiMAX and WLAN Network Design, Optimization and Performance Analysis, John Wiley \& Sons, New York, NY, USA, 1st edition, 2011.

[21] E. K. Tameh and A. R. Nix, "Use of measurement data to analyze the performance of rooftop diffraction and foliage loss algorithms in a 3-D integrated urban/rural propagation model," in Proceedings of the 48th IEEE Vehicular Technology Conference (VTC '98), vol. 1, pp. 303-307, May 1998.

[22] Z. Wang, E. K. Tameh, and A. R. Nix, "Joint shadowing process in urban peer-to-peer radio channels," IEEE Transactions on Vehicular Technology, vol. 57, no. 1, pp. 52-64, 2008.

[23] E. K. Tameh, A. R. Nix, and M. A. Beach, "3-D integrated macro and microcellular propagation model, based on the use of photogrammetric terrain and building data," in Proceedings of the 47th IEEE Vehicular Technology Conference, vol. 3, pp. 19571961, May 1997.

[24] E. K. Tameh and A. R. Nix, "A mixed-cell propagation model for interference prediction in a UMTS network," in Proceedings of the IEEE VTS 53rd Vehicular Technology Conference (VTS SPRING '01), vol. 1, pp. 409-413, May 2001.

[25] E. Mellios, A. R. Nix, and G. S. Hilton, "Ray-tracing urban picocell 3D propagation statistics for LTE heterogeneous networks," in Proceedings of the 7th European Conference on Antennas and Propagation (EuCAP '13), April 2013.

[26] E. Mellios, A. R. Nix, and G. S. Hilton, "Ray-tracing urban macrocell propagation statistics and comparison with WINNER II/+ measurements and models," in Proceedings of the Loughborough Antennas \& Propagation Conference (LAPC '12), pp. 1-4, 2012.

[27] Y. Q. Bian, A. R. Nix, E. K. Tameh, and J. P. McGeehan, "MIMOOFDM WLAN architectures, area coverage, and link adaptation for urban hotspots," IEEE Transactions on Vehicular Technology, vol. 57, no. 4, pp. 2364-2374, 2008.

[28] M. Steinbauer, A. F. Molisch, and E. Bonek, "The doubledirectional radio channel," IEEE Antennas and Propagation Magazine, vol. 43, no. 4, pp. 51-63, 2001. 

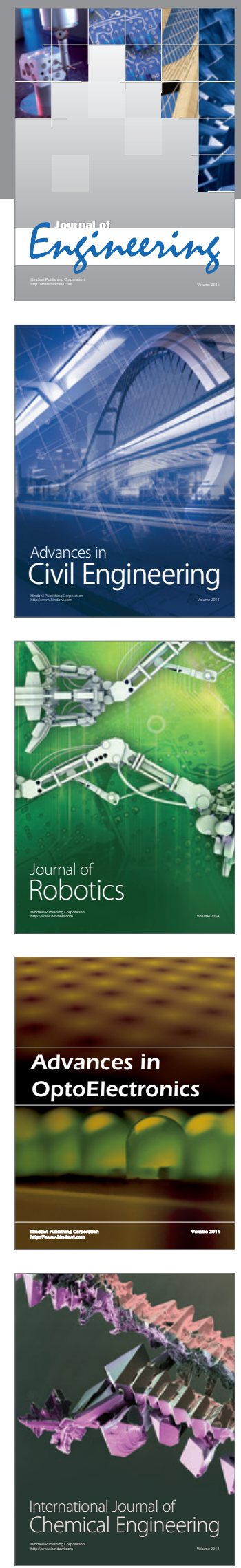

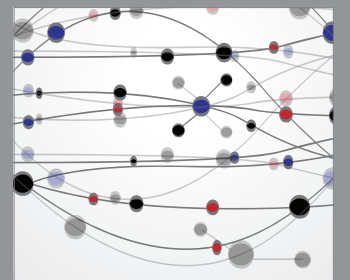

The Scientific World Journal
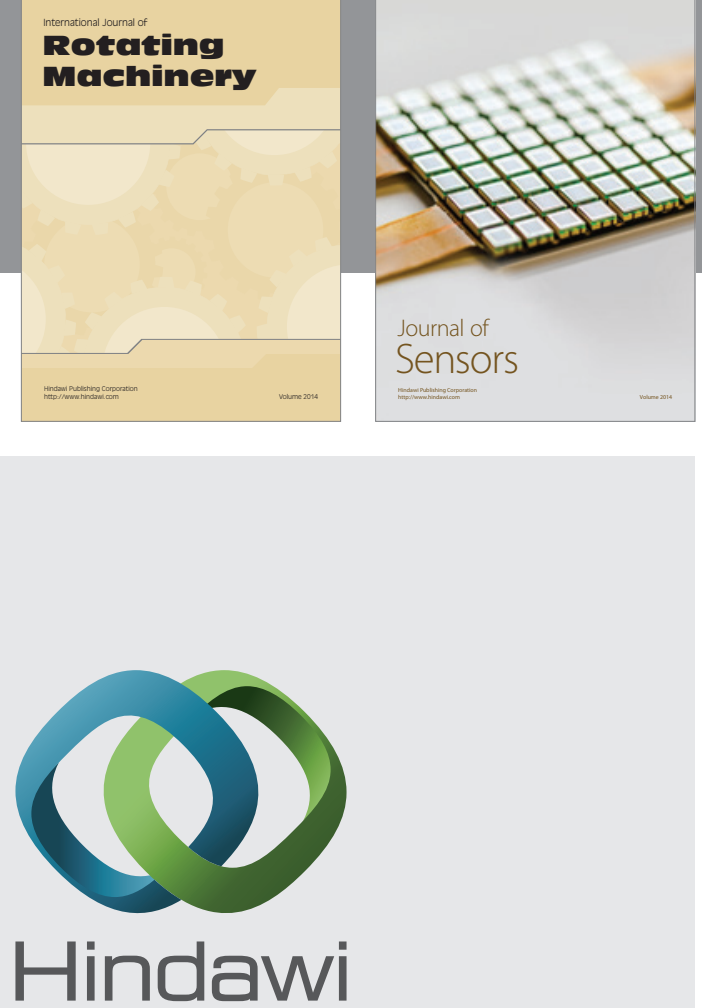

Submit your manuscripts at http://www.hindawi.com
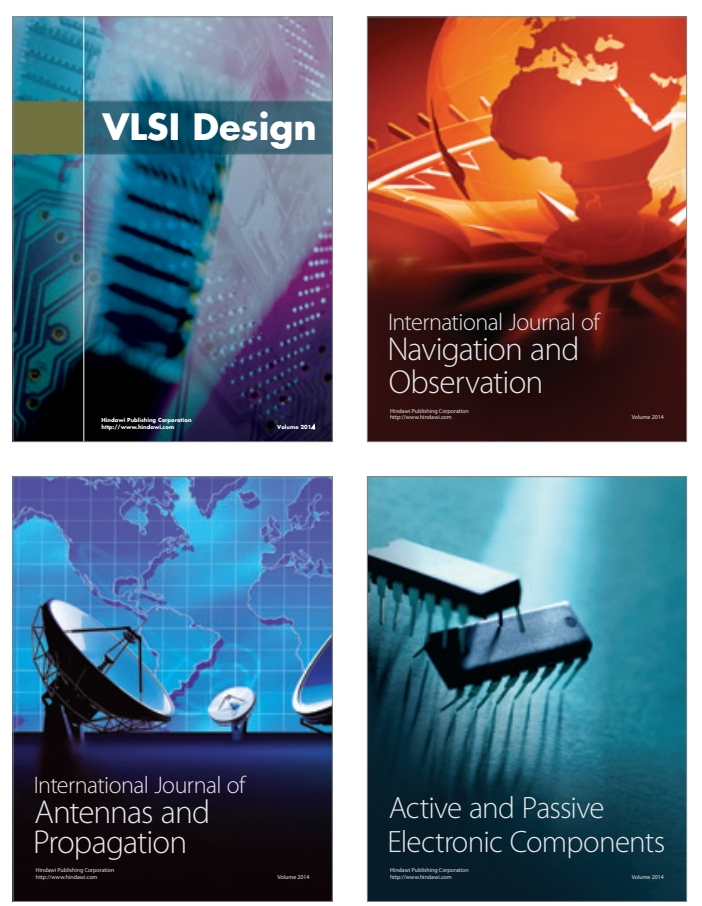
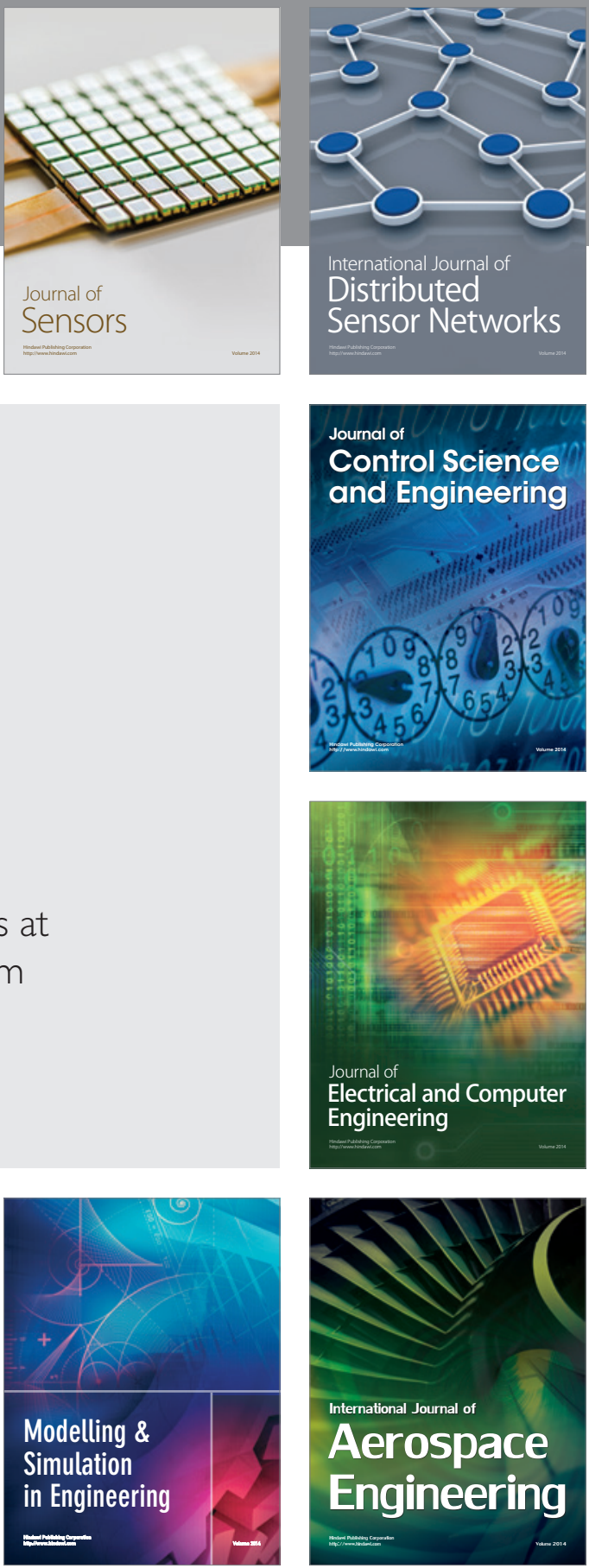

Journal of

Control Science

and Engineering
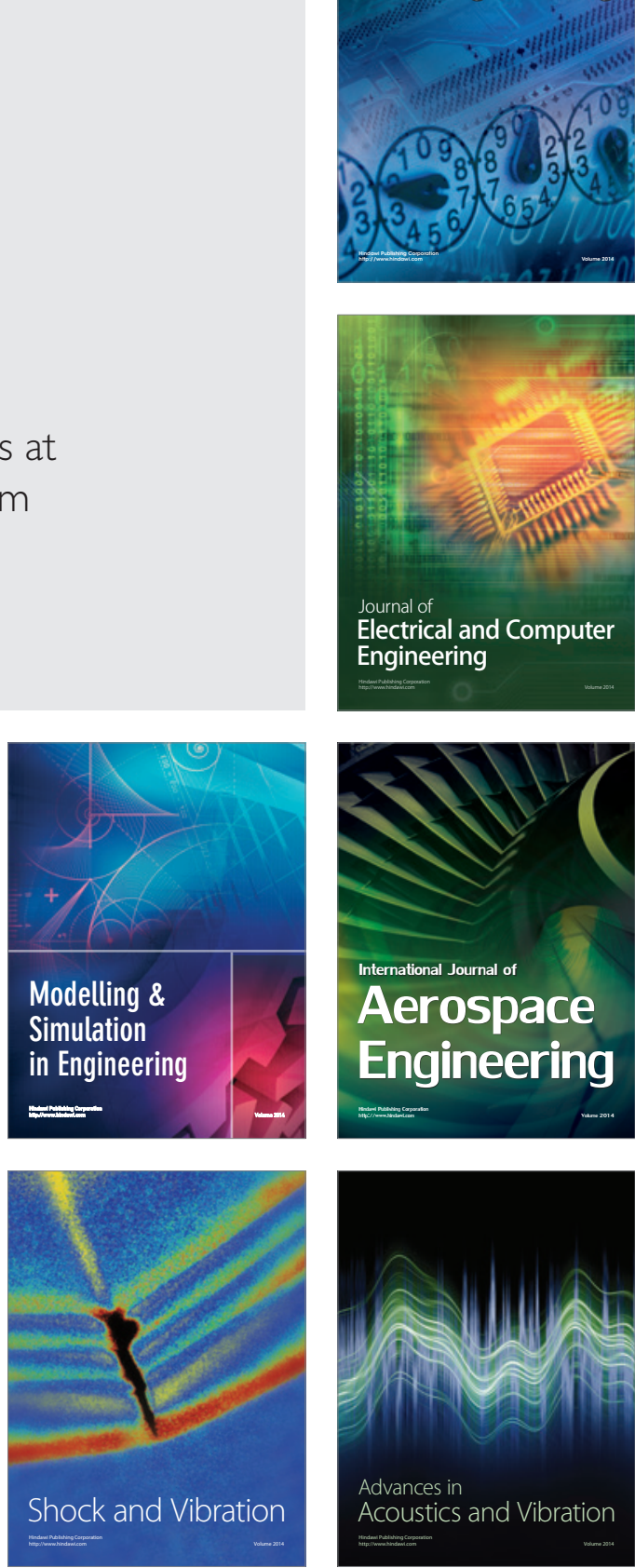